\title{
DIAGONAL DEFECT MEASURES, ADHESION DYNAMICS AND EULER EQUATION *
}

\author{
FRÉDÉRIC POUPAUD ${ }^{\dagger}$
}

\begin{abstract}
This paper is concerned with the existence and the stability of global solutions, with concentrations, for two systems of Partial Differential Equations. The first one is a system modeling adhesion dynamics, the second one is the incompressible Euler equations in vorticity form, with vortex points of distinguished sign. The results are obtained in two space dimension. In order to study the concentrations effects, defect measures for sequences of tensor products of measures are introduced.
\end{abstract}

1. Introduction. The goal of this paper is to study concentration effects in P.D.E. We focus on two systems of equation of physics. The first one is the classical incompressible Euler equation in vorticity formulation. In the 2 -space dimensional setting, this system of equations reads

$$
\begin{array}{r}
\frac{\partial}{\partial t} \omega+\frac{\partial}{\partial x_{1}}\left(u_{1} \omega\right)+\frac{\partial}{\partial x_{2}}\left(u_{2} \omega\right)=0, \quad t \in \mathbb{R}, x \in \mathbb{R}^{2}, \\
\omega=\frac{\partial}{\partial x_{1}} u_{2}-\frac{\partial}{\partial x_{2}} u_{1}, \quad \frac{\partial}{\partial x_{1}} u_{1}+\frac{\partial}{\partial x_{2}} u_{2}=0 \quad t \in \mathbb{R}, x \in \mathbb{R}^{2} .
\end{array}
$$

The scalar $\omega(t, x)$ is the vorticity and $u(t, x)=\left(u_{1}(t, x), u_{2}(t, x)\right)$ is the velocity of the fluid at time $t$ and position $x$. This system of equations is one of the master model for fluid dynamics. It has been intensively studied and we refer to the books of J.-Y. Chemin, [7], C. Marchioro and M. Pulvirenti, [25], or of P.L. Lions, [22], for an excellent survey and a lot of new mathematical results in this field. We only mention that the widest class for which a global existence theorem is known is the class of vorticity with a non negative part which is a bounded measure and a non positive part which is an integrable function and which correspond to a velocity whose square is locally integrable. This famous result is due to J.M. Delort, [9], and simplified proofs can be found in $[14,24,29]$. One of the key ingredient in Delort's proof is that there is no point concentration in space for the vorticity. By essence, the case of vortex points which correspond to the case where there are delta masses in space, for the vorticity, is not considered in these works. However vortex points have a great physical interest in addition to a numerical interest for particles methods, see [25]. One of our result is the existence and stability of generalized weak solution with defect measure for vortex points of distinguished sign, cf Theorems 4.3 and 4.4 .

The other system we want to study, is a model for adhesion dynamics which reads

$$
\begin{array}{r}
\frac{\partial}{\partial t} \rho+\frac{\partial}{\partial x_{1}}\left(u_{1} \rho\right)+\frac{\partial}{\partial x_{2}}\left(u_{2} \rho\right)=0, \quad t \in \mathbb{R}, x \in \mathbb{R}^{2}, \\
\rho=-\frac{\partial}{\partial x_{1}} u_{1}-\frac{\partial}{\partial x_{2}} u_{2}, \quad \frac{\partial}{\partial x_{2}} u_{1}-\frac{\partial}{\partial x_{1}} u_{2}=0 \quad t \in \mathbb{R}, x \in \mathbb{R}^{2} .
\end{array}
$$

The scalar $\rho(t, x)$ is a concentration of particles and $u(t, x)=\left(u_{1}(t, x), u_{2}(t, x)\right)$ is the velocity of the particles at time $t$ and position $x$. This system is in a way orthogonal

\footnotetext{
${ }^{*}$ Received February 18, 2002; accepted for publication December 27, 2002. This work was partially supported by TMR-European Union, contract ERB FMBX-CT97-0157.

${ }^{\dagger}$ Laboratoire J. A. Dieudonné, U.M.R. 6621 C.N.R.S., Université de Nice, Parc Valrose, F-06108 Nice Cedex 2, France (poupaud@unice.fr).
} 
to the Euler system. For instance it can be easily seen that the velocity solves

$$
\frac{\partial}{\partial t} u+u \operatorname{div}(u)=-\nabla^{\perp} p, \quad \operatorname{curl}(u)=0
$$

for a scalar pressure $p, \operatorname{indeed} \operatorname{div}\left(\frac{\partial}{\partial t} u+u \operatorname{div}(u)\right)=0$. Contrarily to Euler equations, $L^{p}$-norms of the vorticity are not conserved quantities. Indeed we have

$$
\frac{D}{D t} \rho \stackrel{\text { def }}{=} \frac{\partial}{\partial t} \rho+u \cdot \nabla \rho=\rho^{2} .
$$

This Ricatti equation shows that there is a blow up of the $L^{\infty}$-norm of the concentration. Actually it can be shown that there is always appearance of delta masses in space, for finite time, despite of the fact the initial data is smooth, cf Proposition 3.2. As for vortex points, we obtain for this adhesion dynamics model a global existence and stability result of generalized solutions with defect measures, cf Theorem 3.2. The model as an obvious generalization in any dimension which reads

$$
\frac{\partial}{\partial t} \rho+\operatorname{div}_{x}(\rho u)=0, \quad u=\nabla_{x} \Phi, \quad-\Delta_{x} \Phi=\rho .
$$

The problem of global existence in dimension higher than 2 is completely open.

Since this system is much less classical than Euler equations, let us spent some lines to give the physics motivations to study it. First, we point out that it has a lot of connections with the equations of pressure-less gases which model sticky particles. Actually, it can be seen as a multi-dimensional generalization of sticky particles models. Pressure-less gases equations has been studied in different contexts, first regarding the properties of solutions associate to the stationary problem ( $\rho$ does not depend of $t$ ). In this direction we mention the work of H. A. Herrero, B. Medina and J. J. L. Velázquez [17] proving blow-up for the solution and the work of P. Constantin, A. J. Majda and E. Tabak [8] as an example of fluid where formation of strong fronts appears. In one dimension the problem of existence and uniqueness for the time dependent pressure-less gases system has been studied by F. Bouchut and F. James in [2], by W. E, Y. Rykov and Y. Sinai in [13] and by Y. Brenier and E. Grenier in [6], who solved the problem by showing its equivalence with the Cauchy problem for scalar conservation laws with general fluxes and monotonic initial conditions. A probabilistic interpretation of sticky particles model is also given by A. Dermoune in [10]. Recently, Y. Brenier [5] has proposed a new multi-dimensional model where the potential $\Phi$ solves the fully non linear Monge Ampère equation:

$$
\operatorname{det}\left(\frac{\partial^{2}}{\partial x_{i} \partial x_{j}} \Phi\right)=\rho
$$

instead of the Poisson equation. It allows to introduce a weak Lagrangian formulation of the problem. For this formulation, Y. Brenier proves the existence and the stability of some global in time weak solutions. For this "Monge Ampère" flow, the particles move straight with constant velocities until concentrations occur. This method does not seem to apply, in the present context.

From the Physics view point, the model of adhesion dynamics we study, can be justified as follows. If we consider particles moving in a thermal bath and interacting 
each other via Newton attractive forces, they can be modeled by the Vlasov-PoissonFokker-Planck system (VPFP in short). When the friction forces due to the thermal bath and the gravitational forces are large and of the same order of magnitude, we end up with the following equations for the distribution function $f_{\epsilon}(t, x, v) \geq 0$ and for the potential $\Phi_{\epsilon}$ :

$$
\begin{array}{r}
\epsilon\left(\frac{\partial f_{\epsilon}}{\partial t}+v \cdot \nabla_{x} f_{\epsilon}\right)+\left(\nabla_{x} \Phi_{\epsilon} \cdot \nabla_{v}\right) f_{\epsilon}=L\left(f_{\epsilon}\right), \\
L\left(f_{\epsilon}\right) \stackrel{\text { def }}{=} \Delta_{v} f_{\epsilon}+\operatorname{div}_{v}\left(v f_{\epsilon}\right)=\operatorname{div}_{v}\left(e^{-\frac{v^{2}}{2}} \nabla_{v}\left(e^{\frac{v^{2}}{2}} f_{\epsilon}\right)\right), \\
\Phi_{\epsilon}=\Gamma_{N} * \rho_{\epsilon}, \quad \rho_{\epsilon}=\int_{\mathbb{R}^{N}} f_{\epsilon} d v
\end{array}
$$

where $\epsilon$ is a small parameter. The kernel $\Gamma_{N}$ is the fundamental solution of $-\Delta$ in $\mathbb{R}^{N}$, which is defined by

$$
\Gamma_{N}(x)= \begin{cases}-\frac{1}{2}|x| & \text { if } N=1 \\ -\frac{1}{2 \pi} \ln |x| & \text { if } N=2 \\ \frac{1}{4 \pi} \frac{1}{|x|} & \text { if } N=3 \ldots\end{cases}
$$

Such a scaling has been first introduced in [27] for semiconductors modeling. In [26] the limit $\epsilon \rightarrow 0$ is performed for the (VPFP) system. The particles concentration $\rho=\lim _{\epsilon \rightarrow 0} \rho_{\epsilon}$ satisfies the limit system

$$
\begin{gathered}
\frac{\partial}{\partial t} \rho(t, x)+\operatorname{div}_{x}(\rho u)=0, \quad t \geq 0, \quad x \in \mathbb{R}^{N}, \\
u \stackrel{\text { def }}{=} \nabla_{x} \Phi(t, x)=\nabla_{x} \Gamma_{N} *_{x} \rho(t, x) .
\end{gathered}
$$

The proof is complete in one space dimension and the velocity $u=-\frac{\partial}{\partial x} \Phi$ is shown to be the unique Kruzkov entropy solution of the Hopf Burgers equation

$$
\frac{\partial}{\partial t} u-\frac{\partial}{\partial x}\left(\frac{u^{2}}{2}\right)=0
$$

Since shocks appear in finite time, it shows the occurrence of concentration (gravitational collapse) for the concentration $\rho=-\frac{\partial}{\partial x} u$. It follows that in the multidimensional case, this system admits classical solutions only for short times. We have ([26])

THEOREM 1.1. If $\rho_{0} \in L^{1}\left(\mathbb{R}^{N}\right) \cap W^{1, \infty}\left(\mathbb{R}^{N}\right)$, then there exists a unique strong solution $(\rho, u)$ of the system (1)-(2) with initial data $\rho_{0}$ which verifies

$$
\left\{\begin{array}{l}
\rho \in W^{1, \infty}\left(\left[0, T^{\prime}\right] \times \mathbb{R}^{N}\right), \\
u \in L^{\infty}\left(0, T^{\prime} ; W^{1, \infty}\left(\mathbb{R}^{N}\right)^{N}\right),
\end{array}\right.
$$

where

$$
0<T^{\prime}<T^{*}=\frac{1}{\left\|\rho_{0}\right\|_{L^{\infty}\left(\mathbb{R}^{N}\right)}} .
$$

If $\rho_{0} \in L^{1}\left(\mathbb{R}^{N}\right) \cap L^{\infty}\left(\mathbb{R}^{N}\right)$ and its first order moment is bounded, then there exists a unique weak solution $(\rho, u)$ to the problem (1)-(2) with initial data $\rho_{0}$ such that $\rho \in L^{\infty}\left(0, T^{\prime} ; L^{1} \cap L^{\infty}\left(\mathbb{R}^{N}\right)\right)$. 
An other field where such models arise is chemo-taxis. One of the basic model was introduced by Keller and Segel in [21]. The corresponding equations read

$$
\begin{gathered}
\frac{\partial}{\partial t} \rho+\chi \nabla(\rho \nabla \Phi)-\delta \Delta \rho=0, \\
\frac{\partial}{\partial t} \Phi-\gamma \Delta \Phi=\beta \rho-\mu \Phi .
\end{gathered}
$$

The positive parameters, $\chi, \delta, \gamma, \beta, \mu$, are biological constants. This system has been studied in different asymptotic regimes. A particular interest is the occurrence of blow up (collapse) of the solution. We refer to the work of Jäger and Luckhaus, [20], or to the paper of Herrero, Velazquez, [18] for example of such results. We also mention the work of Rascle, Ziti [28], see also [32], for a study of self-similar solutions in some variants of the Keller-Segel model. Nothing is known about solutions after collapse and the present work can be seen as a step in this direction. Indeed if we assume that the coefficients of diffusion satisfy $\delta<<\gamma$, we put $\delta=\varepsilon \gamma$ and we rescale the equation according to $\rho \rightarrow R \rho, \Phi \rightarrow V \Phi, t \rightarrow T t$ and $x \rightarrow L x$. Then the choice

$$
T=\sqrt{\varepsilon} \frac{1}{\mu}, L=\sqrt{\varepsilon} \sqrt{\frac{\gamma}{\mu}}, V=\sqrt{\varepsilon} \frac{\gamma}{\chi}, R=\frac{1}{\varepsilon} \frac{\mu V}{\beta},
$$

which is a zoom in time space close to a blow up of a solution, leads to the scaled system

$$
\begin{array}{r}
\frac{\partial}{\partial t} \rho+\nabla(\rho \nabla \Phi)-\sqrt{\varepsilon} \Delta \rho=0, \\
\sqrt{\varepsilon} \frac{\partial}{\partial t} \Phi-\Delta \Phi=\rho-\varepsilon \Phi .
\end{array}
$$

At least formally, we recover the system under study in this paper in the limit $\varepsilon \rightarrow 0$. We point out that Proposition 3.2 gives an upper bound for the collapse time.

Let us focus now on the mathematical techniques we use in this work. The approach of [26] consist in defining a generalized product which gives sense to the product $\rho u$. The skew property of the kernel $K_{N}=\nabla_{x} \Gamma_{N}$ gives rise to the following

Definition 1.1. Let $N=1$ or 2 be the space dimension. Let us define

$$
\begin{array}{ll}
\forall x \in \mathbb{R} \backslash\{0\}, \quad K_{1}(x)=-\frac{1}{2} \operatorname{sign}(x), \quad K_{1}(0)=0, \\
\forall x \in \mathbb{R}^{2} \backslash\{0\}, \quad K_{2}(x)=-\frac{1}{2 \pi} \frac{x}{|x|^{2}}, \quad K_{2}(0)=0 .
\end{array}
$$

Let $\mu \in \mathcal{M}_{1}\left(\mathbb{R}^{N}\right)$ and $v$ be such that $v=K_{N} * \mu,\left(-\Delta_{x} \Psi=\mu, v=\nabla_{x} \Psi\right)$. Then, for every $\varphi \in C_{b}^{1}\left(\mathbb{R}^{N}\right)$ we define

$$
\int_{\mathbb{R}}(v \mu) \varphi d x \stackrel{\text { def }}{=} \int_{\mathbb{R}^{N}} \int_{\mathbb{R}^{N}} K_{N}(x-y)\left(\frac{\varphi(x)-\varphi(y)}{2}\right) \mu(y) \mu(x) d x d y,
$$

which extends the regular case in a natural way.

We first remark that for Euler equations in two space dimension, the Biot Savart law reads $u=K_{2}{ }^{\perp} * \omega$ and Definition 1.1 can be used for the product $u \omega$ 
with $K_{2}$ replaced by $K_{2}^{\perp}$. For $N=1$, the function $K_{1}(x-y)\left(\frac{\varphi(x)-\varphi(y)}{2}\right)$ is continuous. It is an essential argument used in [26] to obtained the weak stability of this definition and the high field limit of the VPFP system. For $N=2$, this function is still bounded but no more continuous on the diagonal $D=\left\{(x, x) / x \in \mathbb{R}^{2}\right\} \subset \mathbb{R}^{4}$. Actually this approach is this one used by J.-M. Delort to obtain his famous result on vortex sheets, [9]. The point is that the $H^{-1}$ estimate of the vortex field for the incompressible Euler equation prevents of the appearance of concentration. It is easy to check that if $\mu$ has no atomic part then $\mu \otimes \mu$ does not charge the diagonal. In our context the measure has an atomic part. The Definition 1.1 makes still sense (by definition of $K_{2}$ the function vanishes on the diagonal) but it is no more stable for the weak topology of measures. The goal of this paper is to prove that up to a defect measure which is localized on the atomic support of the measure $\mu$ we can recover weak stability. The Definition 1.1 being relaxed in such a way, it is then possible to obtained a global existence and the stability of solutions for the system (1)-(2) in two space dimension as well as the existence of weak solution for non negative vortex points.

The spaces $L^{p}, H^{m}, W^{m, p}$ are the usual Sobolev spaces. The set $C^{p}$ is the set of $p$-times continuously differentiable functions, $C_{b}^{p}$ the subspace of functions with all derivatives, up to order $p$, which are bounded, $C_{c}^{p}$ the subspace of functions of $C^{p}$ with compact support.

We denote by $B_{r}$ the ball of radius $r$ of $\mathbb{R}^{N}$.

The space $\mathcal{M}\left(\mathbb{R}^{N}\right)$ is the space of Radon measures, $\mathcal{M}_{1}\left(\mathbb{R}^{N}\right)$ is the space of bounded Radon measures and $\mathcal{M}_{1}^{+}\left(\mathbb{R}^{N}\right) \subset \mathcal{M}\left(\mathbb{R}^{N}\right)$ is the subset of non negative bounded measures. In the following we will have to deal with measures $\mu(t)$ on $\mathbb{R}^{N}$ which depend continuously on the parameter $t \in I$, where $I$ is an open interval of $\mathbb{R}$. These measures can also be considered as distributions on $I \times \mathbb{R}^{N}$. For $\psi \in C_{c}^{0}\left(\mathbb{R}^{N}\right)$ we denote by

$$
\int_{\mathbb{R}^{N}} \psi(x) \mu(t, x) d x
$$

the action of the measure $\mu(t)$ on $\psi$. For $\psi \in C_{c}^{0}\left(I \times \mathbb{R}^{N}\right)$ we use the notation

$$
\int_{I} \int_{\mathbb{R}^{N}} \psi(t, x) \mu(t, x) d x d t=\int_{I}\left(\int_{\mathbb{R}^{N}} \psi(t, x) \mu(t, x) d x\right) d t
$$

which defines the measure $\mu$ on $I \times \mathbb{R}^{N}$.

The next Section is devoted to the definition and properties of diagonal defect measures. In Section 3, we apply the tools developed in Section 2 to the study of adhesion dynamics in dimension 2. We obtain an existence and stability results in Theorem 3.2 and qualitative properties of solutions in Proposition 3.2. Finally Section 4 is concerned with Euler equations in two space dimension. We obtain a global existence result for an initial non negative vorticity with non vanishing atomic parts in Theorem 4.3. This result can be generalized to perturbations in $L^{p}\left(\mathbb{R}^{2}\right)$, $p>2$ of the initial vorticity, cf Theorem 4.4.

2. Defect measures of tensor products. Let $\mu^{n}$ be a sequence of Borelian measures of $\mathbb{R}^{N}$ and $\psi$ be a real test function on $\mathbb{R}^{2 N}$ which is Borelian and bounded. We assume that $\psi$ is continuous except on the diagonal $D:=\left\{(x, x) / x \in \mathbb{R}^{N}\right\} \subset \mathbb{R}^{2 N}$. 
The aim of this Section is to characterize the limit of quantities like

$$
\int_{\mathbb{R}^{2 N}} \psi(x, y) \mu^{n}(x) \mu^{n}(y) d x d y
$$

We first introduce some notations.

Definition 2.2. Let $\mu$ be a Borelian measure of $\mathbb{R}^{N}$. We denote by $S_{a t}(\mu)$ the atomic support of $\mu$. It is a countably set of points of $\mathbb{R}^{N}$.

Consider now $m(x, y)=\mu(x) \otimes \mu(y)$. We immediately check from the definition that we have $S_{a t}(m)=\left\{(a, b), a, b \in S_{a t}(\mu)\right\}$. Let $\chi_{D}$ be the characteristic function of the diagonal. Then by Fubini theorem we have

$$
\mu \otimes \mu(x, y)=\left(1-\chi_{D}\right) \mu \otimes \mu(x, y)+\sum_{a \in S_{a t}(\mu)}(\mu(\{a\}))^{2} \delta(x-a) \otimes \delta(y-a)
$$

We recall that a sequence $\mu^{n} \in \mathcal{M}_{1}\left(\mathbb{R}^{N}\right)$ converges vaguely to $\mu \in \mathcal{M}_{1}\left(\mathbb{R}^{N}\right)$ if it converges in $\mathcal{M}_{1}\left(\mathbb{R}^{N}\right)$ - weak $*$, that is $\forall \varphi \in C_{c}^{0}\left(\mathbb{R}^{N}\right)$

$$
\int_{\mathbb{R}^{N}} \varphi(x) \mu^{n}(x) d x \rightarrow \int_{\mathbb{R}^{N}} \varphi(x) \mu(x) d x .
$$

For non negative sequences of measures $\mu^{n} \in \mathcal{M}_{1}^{+}\left(\mathbb{R}^{N}\right)$ this convergence can be precised. The sequence converges tightly if moreover $\lim _{n \rightarrow \infty} \mu^{n}\left(\mathbb{R}^{N}\right) \rightarrow \mu\left(\mathbb{R}^{N}\right)$. In this case (6) holds for every bounded continuous function. We also say that a sequence $\mu^{n} \in \mathcal{M}_{1}^{+}\left(\mathbb{R}^{N}\right)$ is tightly bounded if for some $M>0$

$$
\begin{array}{r}
\forall n \geq 1, \mu^{n}\left(\mathbb{R}^{N}\right) \leq M, \\
\sup _{n=1,2, \ldots} \mu^{n}\left(\mathbb{R}^{N} \backslash B_{R}\right) \rightarrow 0 \text { as } R \rightarrow \infty .
\end{array}
$$

The condition (7) is the Prokhorov criterion to ensure that the sequence is compact for the tight topology of measures, see [3] for instance. As a consequence, a sequence of non negative measures which vaguely converges is tightly convergent if and only if it is tightly bounded. We recall the following classical result.

LEMMA 2.1. Let $\left(\mu^{n}\right)_{n \geq 1}$ be a sequence of $\mathcal{M}_{1}^{+}\left(\mathbb{R}^{N}\right)$ which converges toward $\mu$ vaguely. Let $F$ be a subset of $\mathbb{R}^{N}$ such that $\mu(F)=0$. Then for every bounded, compactly supported, Borelian function $\varphi$ on $\mathbb{R}^{N}$ which is continuous at every points of $\mathbb{R}^{N} \backslash F$ we have

$$
\int \varphi(x) \mu^{n}(x) d x \rightarrow \int \varphi(x) \mu(x) d x
$$

This Lemma can be found in the book of L. Schwartz, [30]. It is one of the key point of the work of J.-M. Delort on vortex sheets, [9]. Proofs can be found in [30] (Theorems 62-63, chapter IV, paragraph 6) and in $[9,14]$ or $[29]$ in the case where $F$ is closed. We point out that it is trivially false for signed measures as showed by the following example

$$
F=\{0\}, \quad \mu^{n}=\delta(x-1 / n)-\delta(x+1 / n), \quad \mu=0, \quad \varphi(x)=\operatorname{sign}(x) .
$$


The main idea of this Section is the following. Assume that $\mu(F) \neq 0$ and that the discontinuity of the test functions $\varphi$ on $F$ are specified. Then the limit sets of the sequence $\int \varphi(x) \mu^{n}(x) d x$ can be characterized by introducing defect measures.

We introduce now for $i, j=1, . . N$, the Borelian functions

$$
\begin{array}{r}
\forall x \in \mathbb{R}^{N} \backslash\{0\}, \mathcal{N}_{i, j}(x)=\frac{x_{i} x_{j}}{|x|^{2}}, \\
\mathcal{N}_{i, j}(0)=0 .
\end{array}
$$

We denote $\mathcal{N}:=\left(\mathcal{N}_{i, j}\right)_{i, j=1, . . N}$ the corresponding matrix valued function. We have that

$$
\begin{array}{r}
\forall \xi \in \mathbb{R}^{N}, \forall x \in \mathbb{R}^{N} \backslash\{0\}, \quad \mathcal{N}(x) . \xi \cdot \xi=\left(\frac{x \cdot \xi}{|x|}\right)^{2} \geq 0, \\
\forall x \in \mathbb{R}^{N} \backslash\{0\}, \quad \operatorname{tr}(\mathcal{N}(x))=1 .
\end{array}
$$

Therefore $\mathcal{N}$ is a non negative symmetric matrix valued function and its trace is bounded by 1 . We will have to introduce regularization sequences for the functions $\mathcal{N}_{i, j}$.

Definition 2.3. A sequence $\mathcal{N}^{n}:=\left(\mathcal{N}_{i, j}^{n}\right)_{i, j=1, \ldots N}, n=1,2, \ldots$, of symmetric matrix valued functions on $\mathbb{R}^{N}$ is said to be an admissible approximation sequence of the function $\mathcal{N}$ if and only if it satisfies for some real sequence $\varepsilon^{n} \rightarrow 0$

$$
\begin{aligned}
& \text { (i) } \forall r>0, \mathcal{N}^{n}(x) \rightarrow \mathcal{N}(x) \text { uniformly on } \mathbb{R}^{N} \backslash B_{r} \\
& \text { (ii) } \forall x \in \mathbb{R}^{N}, \operatorname{tr}\left(\mathcal{N}^{n}(x)\right) \leq 1+\varepsilon^{n} \\
& \left(\text { iii } \forall \forall x, \xi \in \mathbb{R}^{N}, \mathcal{N}^{n}(x) . \xi . \xi \geq-\varepsilon^{n}|\xi|^{2}\right.
\end{aligned}
$$

We remark that (iii) implies that $\mathcal{N}^{n}(x)+\varepsilon^{n} \mathcal{I}$, where $\mathcal{I}$ is the $N \times N$ identity matrix, is symmetric and non negative. Therefore condition (ii) implies that the coefficients of the matrix $\mathcal{N}^{n}$ are uniformly bounded. There exists some $M>0$ such that for $i, j=1, . . N$ we have

$$
\forall x \in \mathbb{R}^{N}, \forall n \geq 1,\left|\mathcal{N}_{i, j}^{n}(x)\right| \leq M .
$$

The main result of this Section is the following

Proposition 2.1. Let $\mu^{n} \in \mathcal{M}_{1}^{+}\left(\mathbb{R}^{N}\right), n=1,2, \ldots$, be a tightly bounded sequence and $\mathcal{N}^{n}, n=1,2, \ldots$, an admissible approximation sequence of the function $\mathcal{N}$. Then for some subsequence $n_{k}$ there is a measure $\mu \in \mathcal{M}_{1}^{+}\left(\mathbb{R}^{N}\right)$ and diagonal defect measures $\nu_{i, j} \in \mathcal{M}_{1}\left(\mathbb{R}^{N}\right)$ for $i, j=1, . . N$ such that $\mu^{n_{k}} \rightarrow \mu$ tightly and

$$
\begin{gathered}
\forall \varphi \in C_{b}^{0}\left(\mathbb{R}^{2 N}\right), \quad \int_{\mathbb{R}^{2 N}} \varphi(x, y) \mathcal{N}_{i, j}^{n_{k}}(x-y) \mu^{n_{k}}(x) \mu^{n_{k}}(y) d x d y \rightarrow \\
\int_{\mathbb{R}^{2 N}} \varphi(x, y) \mathcal{N}_{i, j}(x-y) \mu(x) \mu(y) d x d y+\int_{\mathbb{R}^{N}} \varphi(x, x) \nu_{i, j}(x) d x .
\end{gathered}
$$

The matrix value measure $\nu=\left(\nu_{i, j}\right)_{i, j=1, . . N}$ is non negative, symmetric and satisfies

$$
\operatorname{tr}(\nu(x)) \leq \sum_{a \in S_{a t}(\mu)}(\mu(\{a\}))^{2} \delta(x-a) .
$$


REMARK 2.1. If $S_{a t}(\mu)=\emptyset$ then the defect measure $\nu$ vanishes. By this way we recover the result of Lemma 2.1 for the measure $\mu \otimes \mu$ with $F=D$. At least, it shows that, in the particular situation described in the Proposition, the above result is stronger than Lemma 2.1.

Proof. Let $\psi \in C_{b}^{0}\left(\mathbb{R}^{N}\right)$, we define Radon measures, $m_{i, j}^{n}$, by

$$
\int_{\mathbb{R}^{N}} \psi(x) m_{i, j}^{n}(x) d x:=\int_{\mathbb{R}^{2 N}} \psi(x) \mathcal{N}_{i, j}^{n}(x-y) \mu^{n}(x) \mu^{n}(y) d x d y .
$$

In view of (9) we have $\left|m_{i, j}^{n}\right| \leq M \mu^{n}\left(\mathbb{R}^{N}\right) \mu^{n}$, therefore these measures are tightly bounded. For a subsequence, still indexed by $n$ for the sake of legibility, we have

$$
m_{i, j}^{n} \rightarrow m_{i, j}, \quad \mu^{n} \rightarrow \mu, \quad \text { tightly. }
$$

We define

$$
\begin{aligned}
\int_{\mathbb{R}^{N}} \psi(x) \nu_{i, j}(x) d x:= & \int_{\mathbb{R}^{N}} \psi(x) m_{i, j}(x) d x \\
& -\int_{\mathbb{R}^{2 N}} \psi(x) \mathcal{N}_{i, j}(x-y) \mu(x) \mu(y) d x d y .
\end{aligned}
$$

It remains to check that $(10)$ and (11) hold. Let $\varphi \in C_{b}^{0}\left(\mathbb{R}^{2 N}\right)$ be a test function. The uniform bound (9), and the point $(i)$ of Definition 2.3 imply that

$$
\begin{aligned}
\forall R>0, \quad(\varphi(x, y)-\varphi(x, x)) \mathcal{N}_{i, j}^{n}(x-y) \rightarrow & \\
& (\varphi(x, y)-\varphi(x, x)) \mathcal{N}_{i, j}(x-y) \quad \text { uniformly on } B_{R} .
\end{aligned}
$$

The sequence $(\varphi(x, y)-\varphi(x, x)) \mathcal{N}_{i, j}^{n}(x-y)$ is uniformly bounded and the function $(\varphi(x, y)-\varphi(x, x)) \mathcal{N}_{i, j}(x-y)$ is continuous and bounded. Then, the fact that $\mu^{n}(x) \otimes$ $\mu^{n}(y) \rightarrow \mu(x) \otimes \mu(y)$ tightly, yields

$$
\begin{array}{r}
\int_{\mathbb{R}^{2 N}}(\varphi(x, y)-\varphi(x, x)) \mathcal{N}_{i, j}^{n}(x-y) \mu^{n}(x) \mu^{n}(y) d x d y \rightarrow \\
\int_{\mathbb{R}^{2 N}}(\varphi(x, y)-\varphi(x, x)) \mathcal{N}_{i, j}(x-y) \mu(x) \mu(y) d x d y .
\end{array}
$$

Using the definition of $m_{i, j}$ and $\nu_{i, j}$ with $\psi(x)=\varphi(x, x)$ we obtain (10). Let $\xi \in \mathbb{R}^{N}$ be a fixed vector. Let $\varphi$ be a non negative, bounded, continuous function. The point (iii) of Definition 2.3 implies

$$
\begin{array}{r}
\forall x, y \in \mathbb{R}^{N}, \sum_{i, j=1, . . N} \mathcal{N}_{i, j}^{n}(x-y) \xi_{i} \xi_{j} \geq-\varepsilon^{n}|\xi|^{2} \\
\sum_{i, j=1, . . N} \int_{\mathbb{R}^{2 N}} \varphi(x, y) \xi_{i} \xi_{j} \mathcal{N}_{i, j}^{n}(x-y) \mu(x) \mu(y) d x d y \geq-C \varepsilon^{n}
\end{array}
$$

for some positive constant $C$ which does not depend on $n$. Using (10) we obtain that

$$
\begin{array}{r}
\sum_{i, j=1, . . N} \int_{\mathbb{R}^{2 N}} \varphi(x, y) \xi_{i} \xi_{j} \mathcal{N}_{i, j}(x-y) \mu(x) \mu(y) d x d y \\
+\sum_{i, j=1, . . N} \int_{\mathbb{R}^{N}} \varphi(x, x) \xi_{i} \xi_{j} \nu_{i, j}(x) d x \geq 0 .
\end{array}
$$


Let $\eta \in C_{c}^{0}\left(\mathbb{R}^{N}\right)$ satisfying $0 \leq \eta \leq 1, \eta(0)=1$. Let $\psi \in C_{b}^{0}\left(\mathbb{R}^{N}\right)$ be any non negative function. We define $\varphi_{p}(x, y)=\eta(p(x-y)) \psi(x)$. The functions $\varphi_{p}(x, y) \xi_{i} \xi_{j} \mathcal{N}_{i, j}(x-y)$ tend to 0 pointwise and are bounded. Then by dominated convergence, the first term in (12) tends to 0 . The second one is constant, so we obtain

$$
\sum_{i, j=1, . . N} \int_{\mathbb{R}^{N}} \psi(x) \xi_{i} \xi_{j} \nu_{i, j}(x) d x \geq 0 .
$$

It proves that $\nu$ is a non negative symmetric matrix valued measure. From the point (ii) of Definition 2.3 we deduce that for all non negative functions $\varphi \in C_{b}^{0}\left(\mathbb{R}^{N}\right)$.

$$
\begin{array}{r}
\left(1+\varepsilon^{n}\right) \int_{\mathbb{R}^{2 N}} \varphi(x, y) \mu^{n}(x) \mu^{n}(y) d x d y \geq \\
\operatorname{tr}\left(\int_{\mathbb{R}^{2 N}} \varphi(x, y) \mathcal{N}^{n}(x-y) \mu^{n}(x) \mu^{n}(y) d x d y\right) .
\end{array}
$$

Passing to the limit, we get

$$
\begin{aligned}
& \int_{\mathbb{R}^{2 N}} \varphi(x, y) \mu(x) \mu(y) d x d y \\
\geq & \operatorname{tr}\left(\int_{\mathbb{R}^{2 N}} \varphi(x, y) \mathcal{N}(x-y) \mu(x) \mu(y) d x d y\right) \\
& +\operatorname{tr}\left(\int_{\mathbb{R}^{N}} \varphi(x, x) \nu(x) d x\right) \\
\geq & \int_{\mathbb{R}^{2 N}} \varphi\left(1-\chi_{D}\right)(x, y) \mu(x) \mu(y) d x d y \\
& +\operatorname{tr}\left(\int_{\mathbb{R}^{N}} \varphi(x, x) \nu(x) d x\right)
\end{aligned}
$$

where $\chi_{D}$ is the characteristic function of the diagonal. We use (5), in order to conclude that

$$
\sum_{a \in S_{a t}(\mu)} \mu(\{a\})^{2} \varphi(a, a) \geq \operatorname{tr}\left(\int_{\mathbb{R}^{N}} \varphi(x, x) \nu(x) d x\right) .
$$

It ends the proof of Proposition 2.1.

Proposition 2.1 gives a generalization of the tensor product of measures acting on functions which are not continuous on the diagonal. The discontinuity has to be of the same kind as the one of the functions $\mathcal{N}_{i, j}$. A remarkable point is that this generalization is stable for the weak convergence of measures. Indeed we can use Proposition 2.1 with the (constant) sequence $\mathcal{N}^{n}=\mathcal{N}$ which is obviously an admissible approximation. Then we obtain the following result.

Corollary 2.1. Let $\mu^{n} \in \mathcal{M}_{1}^{+}\left(\mathbb{R}^{N}\right)$ be a tightly bounded sequence. Let $\nu^{n}$ be a sequence of non negative matrix valued measures on $\mathbb{R}^{N}$, satisfying

$$
\operatorname{tr}\left(\nu^{n}(x)\right) \leq \sum_{a \in S_{a t}\left(\mu^{n}\right)}\left(\mu^{n}(\{a\})\right)^{2} \delta(x-a) .
$$


Then for some subsequence $n_{k}$ there is a measure $\mu \in \mathcal{M}_{1}^{+}\left(\mathbb{R}^{N}\right)$ and diagonal defect measures $\nu_{i, j} \in \mathcal{M}_{1}\left(\mathbb{R}^{N}\right)$ for $i, j=1, . . N$ such that $\mu^{n_{k}} \rightarrow \mu$ tightly and $\forall \varphi \in C_{b}^{0}\left(\mathbb{R}^{2 N}\right)$ we have

$$
\begin{array}{r}
\int_{\mathbb{R}^{2 N}} \varphi(x, y) \mathcal{N}_{i, j}(x-y) \mu^{n_{k}}(x) \mu^{n_{k}}(y) d x d y+\int_{\mathbb{R}^{N}} \varphi(x, x) \nu_{i, j}^{n_{k}}(x) d x \rightarrow \\
\int_{\mathbb{R}^{2 N}} \varphi(x, y) \mathcal{N}_{i, j}(x-y) \mu(x) \mu(y) d x d y+\int_{\mathbb{R}^{N}} \varphi(x, x) \nu_{i, j}(x) d x
\end{array}
$$

The matrix value measure $\nu=\left(\nu_{i, j}\right)_{i, j=1, . . N}$ is non negative, symmetric and satisfies

$$
\operatorname{tr}(\nu(x)) \leq \sum_{a \in S_{a t}(\mu)}(\mu(\{a\}))^{2} \delta(x-a) .
$$

Proof. We first remark that for any $\xi \in \mathbb{R}^{N}$ the sequence of measures $\nu^{n} . \xi . \xi$ is tightly bounded because $\nu^{n} \cdot \xi . \xi \leq|\xi|^{2} \operatorname{tr}\left(\nu^{n}\right) \leq|\xi|^{2} \mu^{n}\left(\mathbb{R}^{N}\right) \mu^{n}$. It follows that up to a subsequence, we can assume it tightly converges. This fact together with Proposition 2.1 for the sequence $\mathcal{N}^{n}=\mathcal{N}$ yields the existence of the limit measures $\nu_{i, j}$ satisfying (13). The non negativity of $\nu$ is obtained as in the proof of Proposition 2.1. The bound of the trace is also obtained in the same way by remarking that for every non negative test function $\varphi$

$$
\begin{aligned}
& \operatorname{tr}\left(\int_{\mathbb{R}^{2 N}} \varphi(x, y) \mathcal{N}(x-y) \mu^{n_{k}}(x) \mu^{n_{k}}(y) d x d y+\int_{\mathbb{R}^{N}} \varphi(x, x) \nu^{n_{k}}(x) d x\right) \\
= & \int_{\mathbb{R}^{2 N}} \varphi(x, y)\left(1-\chi_{D}(x, y)\right) \mu^{n_{k}}(x) \mu^{n_{k}}(y) d x d y \\
& +\operatorname{tr}\left(\int_{\mathbb{R}^{N}} \varphi(x, x) \nu^{n_{k}}(x) d x\right) \\
\leq & \int_{\mathbb{R}^{2 N}} \varphi(x, y)\left(1-\chi_{D}(x, y)\right) \mu^{n_{k}}(x) \mu^{n_{k}}(y) d x d y \\
& +\sum_{a \in S_{a t}\left(\mu^{n_{k}}\right)} \mu^{n_{k}}(\{a\})^{2} \varphi(a, a) \\
= & \int_{\mathbb{R}^{2 N}} \varphi(x, y) \mu^{n_{k}}(x) \mu^{n_{k}}(y) d x d y,
\end{aligned}
$$

where we have used (5) for the last equality. Passing to the limit in this inequality and arguing as in the proof of the Proposition conclude the proof of the corollary.

We can have similar result for measures depending on parameters. We first introduce some definitions. We denote by $\lambda$ the Lebesgue measure on the real line. Let $I$ be a real interval and $\mu(t), t \in I$ a family of bounded non negative measures which are tightly continuous with respect to $t$. Let $\chi_{D}^{n}, n=1,2, \ldots$ be a sequence of bounded continuous functions which converges pointwise to $\chi_{D}$. The measures $\mu(t) \otimes \mu(t) \chi_{D}^{n}$ are tightly continuous with respect to $t$ and converge tightly, pointwise with respect to $t$, toward $\mu(t) \otimes \mu(t) \chi_{D}=\sum_{a \in S_{a t}(\mu(t))}(\mu(t)(\{a\}))^{2} \delta(x-a) \delta(y-a)$. Therefore this family of measures is measurable with respect to $t$. Then the marginal

$$
\int_{\mathbb{R}^{N}} \mu(t) \otimes \mu(t)(x, y) \chi_{D}(x, y) d y=\sum_{a \in S_{a t}(\mu(t))}(\mu(t)(\{a\}))^{2} \delta(x-a)
$$


is also measurable. Since it is locally bounded (by $\mu\left(t, \mathbb{R}^{2}\right) \mu(t)$ ) it is locally integrable with respect to the Lebesgue measure $\lambda$. We denote by

$$
\sum_{a \in S_{a t}(\mu(t))}(\mu(t)(\{a\}))^{2} \delta(x-a) \lambda(t)
$$

the measure in $\mathcal{M}\left(I \times \mathbb{R}^{2}\right)$ defined by

$$
\psi \in C_{c}^{0}\left(I \times \mathbb{R}^{2}\right) \mapsto \int_{I}\left(\sum_{a \in S_{a t}(\mu(t))}(\mu(t)(\{a\}))^{2} \psi(t, a)\right) d t .
$$

The set of time dependent measures equipped with admissible diagonal defect measures is given as follows.

Definition 2.4. Let I be an interval of $\mathbb{R}$, we define the set of time dependent measures with diagonal defects

$$
\begin{array}{r}
\mathcal{D} \mathcal{M}^{+}\left(I ; \mathbb{R}^{N}\right):=\left\{(\mu, \nu) ; \forall t \in I, \mu(t) \in \mathcal{M}_{1}^{+}\left(\mathbb{R}^{N}\right), \nu \in \mathcal{M}\left(I \times \mathbb{R}^{N}\right)^{N \times N},\right. \\
\mu(t) \text { is a tightly continuous with respect to } t, \\
\nu \text { is a non negative, symmetric, matrix valued measure, } \\
\left.\operatorname{tr}(\nu(t, x)) \leq \sum_{a \in S_{a t}(\mu(t))}(\mu(t)(\{a\}))^{2} \delta(x-a) \lambda(t)\right\} .
\end{array}
$$

We have

Definition 2.5. A sequence $\left(\mu^{n}, \nu^{n}\right) \in \mathcal{D M}^{+}\left(I ; \mathbb{R}^{N}\right), n=1,2, \ldots$ converges in the set of measures with diagonal defects toward $(\mu, \nu) \in \mathcal{D} \mathcal{M}^{+}\left(I ; \mathbb{R}^{N}\right)$ if and only if, for every compact interval $K \subset I, \mu^{n_{k}}(t) \rightarrow \mu(t)$ tightly, uniformly with respect to $t \in K$ and $\forall \varphi \in C_{b}^{0}\left(I \times \mathbb{R}^{2 N}\right)$ with $\operatorname{supp}(\varphi) \subset K \times \mathbb{R}^{2 N}$, we have

$$
\begin{gathered}
\int_{I} \int_{\mathbb{R}^{2 N}} \varphi(t, x, y) \mathcal{N}_{i, j}(x-y) \mu^{n}(t, x) \mu^{n}(t, y) d x d y d t+ \\
\int_{I \times \mathbb{R}^{N}} \varphi(x, x) \nu_{i, j}^{n}(t, x) d x d t \rightarrow \\
\int_{I} \int_{\mathbb{R}^{2 N}} \varphi(t, x, y) \mathcal{N}_{i, j}(x-y) \mu(t, x) \mu(t, y) d x d y d t+ \\
\int_{I \times \mathbb{R}^{N}} \varphi(t, x, x) \nu_{i, j}(t, x) d x d t .
\end{gathered}
$$

We note

$$
\left(\mu^{n}, \nu^{n}\right) \rightarrow(\mu, \nu) \quad \text { d.m. }
$$

Let $\mu^{n}(t) \in \mathcal{M}_{1}^{+}\left(\mathbb{R}^{N}\right), n=1,2, \ldots$ be a sequence of non negative measures depending on a real parameter $t \in I$. The sequence is said to be equicontinuous and locally tightly bounded if and only if for all compact intervals $K \subset I$ there is a positive constant $M$ such that $\forall \psi \in C_{c}^{0}\left(\mathbb{R}^{N}\right)$

$$
\begin{aligned}
& \text { the sequence } t \rightarrow \\
& \quad \int_{\mathbb{R}^{N}} \psi(x) \mu^{n}(t, x) d x \text { is equicontinuous on } I, \\
& \quad \forall t \in K, \quad \mu^{n}(t)\left(\mathbb{R}^{N}\right) \leq M, \\
& \sup _{n=1,2, \ldots} \sup _{t \in K} \int_{|x| \geq R} \mu^{n}(t, x) d x \rightarrow 0, \quad \text { as } R \rightarrow \infty .
\end{aligned}
$$


If it holds, there is a measure $\mu(t)$ tightly continuous with respect to time and a subsequence $n_{k}$ such that $\mu^{n_{k}}(t)$ converges tightly, locally uniformly in time toward $\mu(t)$. It means that $\forall \psi \in C_{b}^{0}\left(\mathbb{R}^{N}\right)$, all compact interval $K \subset I$, we have

$$
\int_{\mathbb{R}^{N}} \psi(x) \mu^{n_{k}}(t, x) d x \rightarrow \int_{\mathbb{R}^{N}} \psi(x) \mu(t, x) d x, \quad \text { uniformly for } t \in K .
$$

The uniform convergence with respect to the parameter is necessary to obtain the convergence of the tensor product. Indeed, we have (see [26] for instance) that if $\mu^{n}(t)$ converges tightly, uniformly in time, toward $\mu(t)$ then the tensor product $\mu^{n}(t) \otimes \mu^{n}(t)$ converges tightly, uniformly in time, toward $\mu(t) \otimes \mu(t)$. This allows to argue as in the proofs of Proposition 2.1, and of Corollary 2.1. We have the following new version of Proposition 2.1.

Corollary 2.2. Let I be a real interval, let $\left(\mu^{n}(t)\right)_{n \geq 1}, t \in I$ be a locally tightly bounded, equicontinuous sequence of measures. Let $\mathcal{N}^{n}$ be an admissible approximation sequence of $\mathcal{N}$. Then for some subsequence $n_{k}$ there is $(\mu, \nu) \in \mathcal{D} \mathcal{M}^{+}\left(I ; \mathbb{R}^{N}\right)$ such that for every compact interval $K \subset I, \mu^{n_{k}}(t) \rightarrow \mu(t)$ tightly, uniformly with respect to $t \in K$ and $\forall \varphi \in C_{b}^{0}\left(I \times \mathbb{R}^{2 N}\right)$ with $\operatorname{supp}(\varphi) \subset K \times \mathbb{R}^{2 N}$

$$
\begin{array}{r}
\int_{I} \int_{\mathbb{R}^{2 N}} \varphi(t, x, y) \mathcal{N}_{i, j}^{n_{k}}(x-y) \mu^{n_{k}}(t, x) \mu^{n_{k}}(t, y) d x d y d t \rightarrow \\
\int_{\mathbb{R}^{2 N}} \varphi(x, y) \mathcal{N}_{i, j}(x-y) \mu(x) \mu(y) d x d y d t+ \\
\int_{I} \int_{\mathbb{R}^{N}} \varphi(x, x) \nu_{i, j}(x) d x d t .
\end{array}
$$

We have also the following compactness result analogous to Corollary 2.1.

Corollary 2.3. Let I be a real interval, let $\left(\mu^{n}, \nu^{n}\right)_{n \geq 1}$ be a sequence of measures in $\mathcal{D M}^{+}\left(I ; \mathbb{R}^{N}\right)$. We assume that $\left(\mu^{n}(t)\right)_{n \geq 1}, t \in I$ is a locally tightly bounded, equicontinuous sequence of measures. Then for some subsequence $n_{k}$ there is

$$
(\mu, \nu) \in \mathcal{D M}^{+}\left(I ; \mathbb{R}^{N}\right)
$$

such that

$$
\left(\mu^{n_{k}}, \nu^{n_{k}}\right) \rightarrow(\mu, \nu) \quad \text { d.m. } \quad \text { as } k \rightarrow \infty .
$$

3. Application to adhesion dynamics. We define

$$
K_{i}(x)=-\frac{1}{2 \pi} \frac{x_{i}}{|x|^{2}}, \text { for } x \in \mathbb{R}^{2} \backslash\{0\}, \quad K_{i}(0)=0, \quad i=1,2 .
$$

The Newton adhesion dynamics is modeled by the following equation for the time dependent measure $\rho$ on $\mathbb{R}^{2}$

$$
\begin{array}{r}
\frac{\partial}{\partial t} \rho+\sum_{i=1,2} \frac{\partial}{\partial x_{i}} j_{i}=0, \quad t>0, x \in \mathbb{R}^{2}, \\
\text { with } j_{i}=\rho u_{i}, \quad u_{i}(t)=K_{i} * \rho(t) .
\end{array}
$$


This equation is completed by a Cauchy data

$$
\rho(0)=\rho_{I}, \quad \rho_{I} \in \mathcal{M}_{1}^{+}\left(\mathbb{R}^{2}\right) .
$$

As explained in the introduction, the property that the functions $K_{i}$ are odd, allows to define the distribution $j_{i}=\rho(t) u_{i}(t)$. We have

$$
\begin{array}{r}
\forall \psi \in C_{c}^{\infty}\left(\mathbb{R}^{2}\right), \quad \int_{\mathbb{R}^{2}} \psi(x) j_{i}(t, x) d x:= \\
\frac{1}{2} \int_{\mathbb{R}^{4}}(\psi(x)-\psi(y)) K_{i}(x-y) \rho(t, x) \rho(t, y) d x d y .
\end{array}
$$

We remark that the function $(\psi(x)-\psi(y)) K_{i}(x-y)$ is equivalent to

$$
-\frac{1}{2 \pi} \sum_{j=1,2} \frac{\partial}{\partial x_{j}} \psi(x) \mathcal{N}_{i, j}(x-y)
$$

in the neighborhood of the diagonal. In particular the functions

$$
L_{i}(\psi)=\frac{1}{2}(\psi(x)-\psi(y)) K_{i}(x-y)+\frac{1}{4 \pi} \sum_{j=1,2} \frac{\partial}{\partial x_{j}} \psi(x) \mathcal{N}_{i, j}(x-y)
$$

are bounded and continuous. We summarize these results in the following Lemma

Lemma 3.2. Let $\mu \in \mathcal{M}_{1}^{+}\left(\mathbb{R}^{2}\right)$ then $\forall \psi \in C_{b}^{1}\left(\mathbb{R}^{2}\right)$ and for $i=1,2$ we have

$$
\begin{aligned}
& \frac{1}{2} \int_{\mathbb{R}^{4}}(\psi(x)-\psi(y)) K_{i}(x-y) \mu(x) \mu(y) d x d y= \\
& \quad \int_{\mathbb{R}^{4}} L_{i}(\psi)(x, y) \mu(x) \mu(y) d x d y \\
& \quad-\frac{1}{4 \pi} \sum_{j=1,2} \int_{\mathbb{R}^{4}} \frac{\partial}{\partial x_{j}} \psi(x) \mathcal{N}_{i, j}(x-y) \mu(x) \mu(y) d x d y
\end{aligned}
$$

where the operators $L_{i} \in \mathcal{L}\left(C_{b}^{1}\left(\mathbb{R}^{2}\right) ; C_{b}^{0}\left(\mathbb{R}^{4}\right)\right)$ are defined in (22).

We recall that by definition $\mathcal{N}_{i, j}(0)=0$, so that the above equality makes sense. However we have seen in the previous Section that it is necessary to introduce diagonal defect measures in order to recover stability for the tight convergence of measures.

Definition 3.6. Let I be a real interval, let $(\rho, \nu) \in \mathcal{D M}^{+}\left(I ; \mathbb{R}^{2}\right)$, then the Newton flux $j(\rho, \nu)=\left(j_{1}(\rho, \nu), j_{2}(\rho, \nu)\right)$ is defined by

$$
\begin{array}{r}
\forall \psi \in C_{c}^{1}\left(I \times \mathbb{R}^{2}\right), \quad \int_{I} \int_{\mathbb{R}^{2}} \psi(t, x) j_{i}(\rho, \nu)(t, x) d x d t= \\
\frac{1}{2} \int_{I} \int_{\mathbb{R}^{4}}(\psi(t, x)-\psi(t, y)) K_{i}(x-y) \rho(t, x) \rho(t, y) d x d y d t \\
-\frac{1}{4 \pi} \sum_{j=1,2} \int_{I} \int_{\mathbb{R}^{2}} \frac{\partial}{\partial x_{j}} \psi(t, x) \nu_{i, j}(t, x) d x d t
\end{array}
$$

A direct consequence of Definition 2.5 and of Lemma 3.2 is the following stability result. 
LEMMA 3.3. Let $I$ be a real interval and $\left(\rho^{n}, \nu^{n}\right) \in \mathcal{D} \mathcal{M}^{+}\left(I ; \mathbb{R}^{2}\right), n=1,2, \ldots$, $(\rho, \nu) \in \mathcal{D} \mathcal{M}^{+}\left(I ; \mathbb{R}^{2}\right)$ such that

$$
\left(\rho^{n}, \nu^{n}\right) \rightarrow(\rho, \nu) \quad \text { d.m. }
$$

then for $i=1,2$ we have

$$
\begin{gathered}
\forall \psi \in C_{c}^{1}\left(I \times \mathbb{R}^{2}\right) \\
\int_{I} \int_{\mathbb{R}^{2}} \psi(t, x) j_{i}\left(\rho^{n}, \nu^{n}\right)(t, x) d x d t \rightarrow \int_{I} \int_{\mathbb{R}^{2}} \psi(t, x) j_{i}(\rho, \nu)(t, x) d x d t .
\end{gathered}
$$

Proof. In view of (24) and using Lemma 3.2 we get

$$
\begin{array}{r}
\forall \psi \in C_{b}^{1}\left(I \times \mathbb{R}^{2}\right), \quad \int_{I} \int_{\mathbb{R}^{2}} \psi(t, x) j_{i}(\rho, \nu)(t, x) d x d t= \\
\frac{1}{2} \int_{I} \int_{\mathbb{R}^{4}} L_{i}(\psi)(x, y) \rho^{n}(t, x) \rho^{n}(t, y) d x d y d t \\
-\frac{1}{4 \pi} \sum_{j=1,2} \int_{I} \int_{\mathbb{R}^{4}} \frac{\partial}{\partial x_{j}} \psi(t, x) \mathcal{N}_{i, j}(x-y) \rho^{n}(t, x) \rho^{n}(t, y) d x d t \\
-\frac{1}{4 \pi} \sum_{j=1,2} \int_{I} \int_{\mathbb{R}^{4}} \frac{\partial}{\partial x_{j}} \psi(t, x) \nu_{i, j}^{n}(t, x) d x d t
\end{array}
$$

The first integral converges because $\rho^{n}(t, x) \rho^{n}(t, y) \rightarrow \rho(t, x) \rho(t, y)$ tightly on $\mathbb{R}^{4}$ uniformly with respect to $t \in K$, for any compact interval $K \subset I$. The last two integrals converge by Definition 2.5.

This continuity result of the Newton flux allows to obtain the stability of generalized solutions to Newton adhesion dynamics.

Definition 3.7. Let $T \in(0,+\infty]$, a couple $(\rho, \nu) \in \mathcal{D} \mathcal{M}^{+}\left([0, T) ; \mathbb{R}^{2}\right)$ is a generalized solution on $[0, T)$ of the Newton adhesion dynamics equation with the Cauchy data $\rho_{I} \in \mathcal{M}_{1}^{+}\left(\mathbb{R}^{2}\right)$ if and only if $\rho(0)=\rho_{I}$ and

$$
\frac{\partial}{\partial t} \rho+\sum_{i=1,2} \frac{\partial}{\partial x_{i}} j_{i}(\rho, \nu)=0
$$

in the sense of distribution on $(0, T) \times \mathbb{R}^{2}$.

Let us remark that if there is no atomic part to the measures $\rho(t), t \in[0, T)$ then the defect measure vanishes and the definition of $j_{i}(\rho, 0)$ coincide with the classical definition (21). The generalized solutions also satisfy the classical conservation law and are stable for the weak convergence of measures.

THEOREM 3.2. We have the following results concerning generalized solutions. Global existence. For all initial data $\rho_{I} \in \mathcal{M}_{1}^{+}\left(\mathbb{R}^{2}\right)$, there is a generalized solution on $[0, \infty)$.

A priori estimates. Let $T \in(0,+\infty]$, and let $(\rho, \nu) \in \mathcal{D} \mathcal{M}^{+}\left([0, T) ; \mathbb{R}^{2}\right)$ be a generalized solution on $[0, T)$ of the Newton adhesion dynamics equation with the Cauchy data $\rho_{I} \in \mathcal{M}_{1}^{+}\left(\mathbb{R}^{2}\right)$. Then we have the conservation law

$$
\forall t \in[0, T), \quad \rho\left(t, \mathbb{R}^{2}\right)=\rho_{I}\left(\mathbb{R}^{2}\right) .
$$


Moreover, we have an a priori tight bound of the solution. There is a universal constant, $C>0$, such that

$$
\forall R>0, \quad \forall t \in[0, T), \quad \rho\left(t, \mathbb{R}^{2} \backslash B_{R}\right) \leq \rho_{I}\left(\mathbb{R}^{2} \backslash B_{R / 2}\right)+C \frac{t}{R^{2}} \rho_{I}\left(\mathbb{R}^{2}\right)^{2} .
$$

If $|x| \rho_{I}$ is assumed to be a bounded measure then the same is true for $|x| \rho(t)$, $t \in[0, T)$ and there is a universal constant $C>0$ such that

$$
\begin{aligned}
& \forall t \in[0, T), \int_{\mathbb{R}^{2}}|x| \rho(t, x) d x \leq \int_{\mathbb{R}^{2}}|x| \rho_{I}(x) d x \\
& \quad+C \rho_{I}\left(\mathbb{R}^{2}\right)\left(1+t \rho_{I}\left(\mathbb{R}^{2}\right)\right) .
\end{aligned}
$$

In this case the mean position of particles defined by

$$
X(t):=\int_{\mathbb{R}^{2}} x \frac{\rho(t, x) d x}{\rho\left(t, \mathbb{R}^{2}\right)}
$$

is conserved

$$
\forall t \in[0, T), \quad X(t)=X_{I}:=\int_{\mathbb{R}^{2}} x \frac{\rho_{I}(x) d x}{\rho_{I}\left(\mathbb{R}^{2}\right)} .
$$

The equicontinuity of generalized solutions can be estimated as follows. For every test function $\psi \in C_{b}^{2}\left(\mathbb{R}^{2}\right)$, there is a positive constant $C(\psi)$ which depends only on the test function such that

$$
\left|\frac{d}{d t} \int_{\mathbb{R}^{2}} \psi(x) \rho(t, x) d x\right| \leq C(\psi) \rho_{I}\left(\mathbb{R}^{2}\right)^{2},
$$

in the distribution sense on $(0, T)$.

It follows that if $\left(\rho^{n}, \nu^{n}\right) \in \mathcal{D} \mathcal{M}^{+}\left([0, T) ; \mathbb{R}^{2}\right), n=1,2, \ldots$ is a sequence of generalized solutions such that $\rho_{I}^{n}=\rho^{n}(t=0)$ is tightly bounded then the sequence $\rho^{n}(t), t \in[0, T)$ is locally tightly uniformly bounded and equicontinuous with respect to time.

Stability. The set of generalized solutions is stable. More precisely let $\left(\rho^{n}, \nu^{n}\right) \in$ $\left.\left.\overline{\mathcal{D} \mathcal{M}^{+}([0}, T\right) ; \mathbb{R}^{2}\right), n=1,2, \ldots$ be a sequence of approximated solutions of Coulomb adhesion dynamics:

$$
\frac{\partial}{\partial t} \rho^{n}+\sum_{i=1,2} \frac{\partial}{\partial x_{i}} j_{i}\left(\rho^{n}, \nu^{n}\right)=S^{n}
$$

with $S^{n} \rightarrow 0$ in the sense of distribution on $(0, T) \times \mathbb{R}^{2}$ as $n \rightarrow \infty$. We assume that the sequence of measures $\left(\rho^{n}(t)\right)_{n \geq 1}$ is locally tightly uniformly bounded and equicontinuous with respect to time. Then all accumulation points of the sequence $\left(\rho^{n}, \nu^{n}\right)$ for the convergence in diagonal defect measures obtained by Corollary 2.3 are generalized solutions of the Newton adhesion dynamics equation.

Proof.

Stability. The stability result is a direct consequence of Lemma 3.3 and of Definition 3.7. 
A priori estimates. We use Definition 3.7 with test functions of the form $\varphi(t, x)=$ $\alpha(t) \psi(x), \alpha \in C_{c}^{\infty}(I), \psi \in C_{c}^{\infty}\left(\mathbb{R}^{2}\right)$. We obtain the following equality in the sense of distribution on $(0, T)$

$$
\begin{array}{r}
\frac{d}{d t} \int_{\mathbb{R}^{2}} \psi(x) \rho(t, x) d x= \\
\frac{1}{2} \sum_{i=1}^{2} \int_{\mathbb{R}^{4}}\left(\frac{\partial}{\partial x_{i}} \psi(x)-\frac{\partial}{\partial x_{i}} \psi(y)\right) K_{i}(x-y) \rho(t, x) \rho(t, y) d x d y \\
-\frac{1}{4 \pi} \sum_{i, j=1}^{2} \int_{\mathbb{R}^{2}} \frac{\partial^{2}}{\partial x_{i} \partial x_{j}} \psi(x) \nu_{i, j}(t, x) d x
\end{array}
$$

Since $\nu$ is symmetric, non negative matrix valued, by Schwartz inequality we have

$$
\begin{aligned}
\left|\nu_{i, j}\right|\left(t, \mathbb{R}^{2}\right) & \leq \sqrt{\nu_{i, i}\left(t, \mathbb{R}^{2}\right) \nu_{j, j}\left(t, \mathbb{R}^{2}\right)} \\
& \leq \operatorname{tr}\left(\nu\left(t, \mathbb{R}^{2}\right) \leq \rho\left(t, \mathbb{R}^{2}\right)^{2},\right.
\end{aligned}
$$

where we have used (15) to obtain the last inequality. If we denote

$$
|\psi|_{2, \infty}:=\sup _{x \in \mathbb{R}^{2}, i, j=1,2}\left|\frac{\partial^{2}}{\partial x_{i} \partial x_{j}} \psi(x)\right|,
$$

we obtain for a universal constant $C>0$

$$
\text { For a.e. } t \in(0, T), \quad\left|\frac{d}{d t} \int_{\mathbb{R}^{2}} \psi(x) \rho(t, x) d x\right| \leq C|\psi|_{2, \infty} \rho\left(t, \mathbb{R}^{2}\right)^{2} \text {. }
$$

We choose $\psi(x)=\beta\left(|x|^{2} / R^{2}\right)$ where $\beta \in C_{c}^{\infty}(\mathbb{R})$ is non negative and equal to 1 in a neighborhood of 0 . We obtain

$$
\left|\frac{d}{d t} \int_{\mathbb{R}^{2}} \beta\left(|x|^{2} / R^{2}\right) \rho(t, x) d x\right| \leq C(\beta) \frac{1}{R^{2}} \rho\left(t, \mathbb{R}^{2}\right)^{2} .
$$

The limit $R \rightarrow \infty$ yields that $\frac{d}{d t} \rho\left(t, \mathbb{R}^{2}\right)=0$ in the sense of distribution on $(0, T)$. Since by definition $\rho\left(t, \mathbb{R}^{2}\right)$ is continuous, in particular at $t=0$, we obtain the conservation law (25). The estimate (30) is a direct consequence of (31) and of (25). An other consequence of (25) is that we also have

$$
\left|\frac{d}{d t} \int_{\mathbb{R}^{2}}\left(1-\beta\left(|x|^{2} / R^{2}\right)\right) \rho(t, x) d x\right| \leq C(\beta) \frac{1}{R^{2}} \rho_{I}\left(\mathbb{R}^{2}\right)^{2} .
$$

We choose $\beta$ such that $0 \leq \beta \leq 1, \beta(r)=1$ for $0 \leq r \leq 1 / 2, \beta(r)=0$ for $r \geq 1$, in order to obtain (26). We can also use (31) with $\psi(x)=\sqrt{1+|x|^{2}} \beta\left(|x|^{2} / R^{2}\right)$. The semi-norm $|\psi|_{2, \infty}$ is bounded independently of $R$. Therefore the limit $R \rightarrow \infty$ gives

$$
\int_{\mathbb{R}^{2}} \sqrt{1+|x|^{2}} \rho(t, x) d x \leq \int_{\mathbb{R}^{2}} \sqrt{1+|x|^{2}} \rho_{I}(x) d x+C t \rho_{I}\left(\mathbb{R}^{2}\right)^{2} .
$$

It leads to (27). Now we can use $\psi(x)=x \beta\left(|x|^{2} / R^{2}\right)$ in (31). We have $|\psi|_{2, \infty} \leq C(\beta) / R$. The limit $R \rightarrow \infty$ leads to the conservation of the first momentum 
$\int_{\mathbb{R}^{2}} x \rho(t, x) d x$ which together with (25) leads to (29).

Let $\rho_{I}^{n} \in \mathcal{M}_{1}^{+}\left(\mathbb{R}^{2}\right), n=1,2, \ldots$, be a tightly bounded sequence of non negative measures. Then it follows from (25), (26) that a corresponding sequence of generalized solutions $\rho^{n}(t), n=1,2, \ldots, t \in[0, T)$ is tightly bounded uniformly with respect to time on every interval $\left[0, T_{0}\right]$ with $T_{0}<T$. Let $\psi \in C_{c}^{0}\left(\mathbb{R}^{2}\right)$, for all $\varepsilon>0$ there exists $\psi_{\varepsilon} \in C_{c}^{\infty}\left(\mathbb{R}^{2}\right)$ such that $\forall x \in \mathbb{R}^{2},\left|\psi(x)-\psi_{\varepsilon}(x)\right| \leq \varepsilon$. A consequence of (30) is that

$$
\begin{aligned}
\forall t, s \in[0, T), & \left|\int_{\mathbb{R}^{2}} \psi(x) \rho^{n}(t, x) d x-\int_{\mathbb{R}^{2}} \psi(x) \rho^{n}(s, x) d x\right| \\
\leq & \left|\int_{\mathbb{R}^{2}}\left(\psi(x)-\psi_{\varepsilon}(x)\right)\left(\rho^{n}(t, x)-\rho^{n}(s, x)\right) d x\right| \\
& +\left|\int_{\mathbb{R}^{2}} \psi_{\varepsilon}(x) \rho^{n}(t, x) d x-\int_{\mathbb{R}^{2}} \psi_{\varepsilon}(x) \rho^{n}(s, x) d x\right|, \\
\leq & 2 \varepsilon \rho_{I}^{n}\left(\mathbb{R}^{2}\right)+C\left(\psi_{\varepsilon}\right) \rho_{I}^{n}\left(\mathbb{R}^{2}\right)^{2}|t-s|, \\
\leq & C\left(\varepsilon+C\left(\psi_{\varepsilon}\right)|t-s|\right),
\end{aligned}
$$

for a constant $C$ independent on $n$. Therefore the sequence is equicontinuous with respect to time.

Global existence. We choose $\beta \in C_{c}^{\infty}(\mathbb{R})$ such that $0 \leq \beta \leq 1, \beta(r)=1$ for $0 \leq r \leq 1 / 2, \beta(r)=0$ for $r \geq 1$. For $n=1,2, \ldots$, let us define $\forall x \in \mathbb{R}^{2}, \gamma^{n}(x)=$ $1-\beta\left(n^{2}|x|^{2}\right), K_{i}^{n}(x)=K_{i}(x) \gamma^{n}(x)$. The functions $K_{i}^{n}$ belong to $C_{b}^{\infty}\left(\mathbb{R}^{2}\right)$. We also regularize the initial data $\rho_{I} \in \mathcal{M}_{1}^{+}\left(\mathbb{R}^{2}\right)$. So, let $\rho_{I}^{n} \in C_{c}^{\infty}\left(\mathbb{R}^{2}\right), n=1,2, \ldots$, be a sequence of non negative functions such that $\rho_{I}^{n} \rightarrow \rho_{I}$ tightly. For $n=1,2, \ldots$, a fixed point method gives the existence and the uniqueness of a smooth solution $\rho^{n}$ of the regularized adhesion dynamics

$$
\begin{aligned}
& \qquad \begin{array}{c}
\frac{\partial}{\partial t} \rho^{n}(t, x)+\sum_{i=1,2} \frac{\partial}{\partial x_{i}} j_{i}^{n}(t, x)=0, \quad t>0, x \in \mathbb{R}^{2}, \\
\text { with } \quad j_{i}^{n}=\rho^{n}\left(K_{i}^{n} *_{x} \rho^{n}\right) \\
\rho^{n}(t=0)=\rho_{I}^{n} .
\end{array}
\end{aligned}
$$

The same arguments as in the previous part of the proof show that $\rho^{n}(t), n=1,2, \ldots$, $t \in[0, \infty)$, is locally uniformly tightly bounded and equicontinuous with respect to time. We also remark that $K_{i}^{n}$ is an odd function. So, as for Lemma 3.2, we obtain

$$
\begin{aligned}
& \forall \varphi \in C_{c}^{\infty}\left((0, \infty) \times \mathbb{R}^{2}\right), \quad \int_{0}^{\infty} \int_{\mathbb{R}^{2}} \varphi(t, x) j_{i}^{n}(t, x) d x d t \\
= & \int_{0}^{\infty} \int_{\mathbb{R}^{4}} L_{i}(\varphi)(t, x, y) \gamma^{n}(x-y) \rho^{n}(t, x) \rho^{n}(t, y) d x d y d t \\
& +\sum_{j=1}^{2} \int_{0}^{\infty} \int_{\mathbb{R}^{4}} \frac{\partial}{\partial x_{j}} \varphi(t, x) \mathcal{N}_{i, j}^{n}(x-y) \rho^{n}(t, x) \rho^{n}(t, y) d x d y d t, \\
& \text { with } \mathcal{N}_{i, j}^{n}(x)=\mathcal{N}_{i, j}(x) \gamma^{n}(x) .
\end{aligned}
$$

The sequence $\mathcal{N}^{n}$ is obviously an admissible approximation of the matrix valued function $\mathcal{N}$ in the sense of Definition 2.3. Then we can use Corollary 2.2 to obtain 
for a subsequence, still labeled by $n$ for the sake of legibility, the existence of a limit $(\rho, \nu) \in \mathcal{D} \mathcal{M}^{+}\left([0, \infty) ; \mathbb{R}^{2}\right)$ such that

$$
\begin{gathered}
\int_{0}^{\infty} \int_{\mathbb{R}^{4}} \frac{\partial}{\partial x_{j}} \varphi(t, x) \mathcal{N}_{i, j}^{n}(x-y) \rho^{n}(t, x) \rho^{n}(t, y) d x d y d t \\
\rightarrow \int_{0}^{\infty} \int_{\mathbb{R}^{4}} \frac{\partial}{\partial x_{j}} \varphi(t, x) \mathcal{N}_{i, j}(x-y) \rho(t, x) \rho(t, y) d x d y d t \\
\quad+\int_{0}^{\infty} \int_{\mathbb{R}^{2}} \frac{\partial}{\partial x_{j}} \varphi(t, x) \nu_{i, j}(t, x) d x d t
\end{gathered}
$$

We remark that $L_{i}(\varphi)$ is uniformly continuous and $\forall(t, x) \in(0, \infty) \times \mathbb{R}^{2}$ we have $L_{i}(\varphi)(t, x, x)=0$. Then we can easily check that

$$
L_{i}(\varphi)(t, x, y) \gamma^{n}(x-y) \rightarrow L_{i}(\varphi)(t, x, y), \quad \text { uniformly on }(0, \infty) \times \mathbb{R}^{4} .
$$

On the other hand, for all $T>0, \rho^{n}(t) \rightarrow \rho(t)$ tightly, uniformly with respect to $t \in[0, T]$. Therefore $\rho^{n}(t, x) \rho^{n}(t, y) \rightarrow \rho(t, x) \rho(t, y)$ tightly, uniformly with respect to $t \in[0, T]$. We conclude that

$$
\begin{array}{r}
\int_{0}^{\infty} \int_{\mathbb{R}^{4}} L_{i}(\varphi)(t, x, y) \gamma^{n}(x-y) \rho^{n}(t, x) \rho^{n}(t, y) d x d y d t \\
\rightarrow \int_{0}^{\infty} \int_{\mathbb{R}^{4}} L_{i}(\varphi)(t, x, y) \rho(t, x) \rho(t, y) d x d y d t
\end{array}
$$

We deduce from (34), (35) and (36) that $j_{i}^{n} \rightarrow j_{i}(\rho, \nu)$ in the distribution sense on $(0, \infty) \times \mathbb{R}^{2}$. It allows to pass to the limit in $(32)$ and in $(33)$. We obtain that $(\rho, \nu)$ is a generalized solution of the adhesion dynamic equation corresponding to the Cauchy data $\rho_{I}$.

In the last Proposition of this Section, our goal is to prove that there is always appearance of an atomic part for generalized solution and to try to characterize the defect measure. We also give the large time behavior of global generalized solutions.

Proposition 3.2. Let $(\rho, \nu) \in \mathcal{D} \mathcal{M}^{+}\left([0, T) ; \mathbb{R}^{2}\right)$ be a generalized solution. Let $S=\left\{(t, a) / t \in[0, T), a \in S_{a t}(\rho(t))\right\}$ be the singular support of the measure $\rho$. If $\Gamma$ is a Borellian set which does not meet $S(\Gamma \cap S=\emptyset)$ or if $\Gamma$ is an Hölder continuous curve, $\Gamma=\left\{(t, Z(t)) / t \in[0, T), Z \in C^{0, \gamma}\left(\mathbb{R} ; \mathbb{R}^{2}\right)\right\}$, with coefficient $\gamma>1 / 2$ then the defect measure $\nu$ does not charge $\Gamma: \int_{\Gamma} \nu(t, x) d x d t=0$.

If $\rho_{I}=\rho(t=0)$ has its second moment bounded, $\int_{\mathbb{R}^{2}}|x|^{2} \rho_{I}(x) d x<\infty$, then the maximal time $T_{0}$ where $\rho(t)$ has no atomic part for almost every $t \in\left[0, T_{0}\right]$ is bounded by

$$
T_{0} \leq \frac{2 \pi}{\rho_{I}\left(\mathbb{R}^{2}\right)^{2}} \int_{\mathbb{R}^{2}}\left|x-X_{I}\right|^{2} \rho(x) d x,
$$

where $X_{I}$ is the mean position of particles defined by (29).

If $(\rho, \nu) \in \mathcal{D M}^{+}\left([0, \infty) ; \mathbb{R}^{2}\right)$ is a global generalized solution then the defect measure $(s, x) \rightarrow \nu(t+s, x)$ tends to 0 tightly as a measure on $[0, \infty) \times \mathbb{R}^{2}$ and $\rho(t) \rightarrow \rho_{I}\left(\mathbb{R}^{2}\right) \delta\left(x-X_{I}\right)$ tightly as $t \rightarrow \infty$. 
Proof. The fact that the defect measure $\nu$ does not charge $\Gamma$, when $\Gamma$ does not meet $S$, is only a consequence of the bound on the trace given in Definition (15). Let now $Z \in C^{0, \gamma}\left(\mathbb{R} ; \mathbb{R}^{2}\right)$. Let $\zeta \in C_{0}^{\infty}(\mathbb{R})$ such that $\int_{\mathbb{R}} \zeta(s) d s=1$. We consider the regularization $Z_{n}=Z * \zeta_{\alpha_{n}}$ with $\zeta_{\alpha_{n}}(t)=\frac{1}{\alpha_{n}} \zeta\left(\frac{t}{\alpha_{n}}\right)$, where $\alpha_{n} \rightarrow 0$ is a sequence to be chosen later on. For $t$ in a compact interval, we have for some constant $C>0$

$$
\begin{aligned}
\left|Z(t)-Z_{n}(t)\right| & \leq \int_{\mathbb{R}}\left|Z\left(t-\alpha_{n} s\right)-Z_{n}(t)\right| \zeta(s) d s \\
& \leq C \alpha_{n}{ }^{\gamma} .
\end{aligned}
$$

For the time derivative of the regularized curve, we have the following estimate

$$
\begin{aligned}
\left|\frac{d}{d t} Z_{n}(t)\right| & =\left|\int_{\mathbb{R}} Z(s) \frac{1}{\alpha_{n}^{2}} \frac{d}{d t} \zeta\left(\frac{t-s}{\alpha_{n}}\right) d s\right| \\
& =\left|\int_{\mathbb{R}}\left(Z\left(t-\alpha_{n} s\right)-Z(t)\right) \frac{1}{\alpha_{n}} \frac{d}{d t} \zeta(s) d s\right| \\
& \leq C \frac{1}{\alpha_{n}^{1-\gamma}} .
\end{aligned}
$$

For $\gamma \in\left(\frac{1}{2}, 1\right)$ we choose $\alpha_{n}=n^{-\delta}$ with $\frac{1}{\gamma}<\delta<\frac{1}{1-\gamma}$. In such a way, we obtain that for any $T_{0}>0$ there is a constant $C>0$ such that

$$
\forall t \in\left[0, T_{0}\right], \quad\left|\frac{1}{n} \frac{d}{d t} Z_{n}(t)\right| \leq C, \quad \frac{1}{n} \frac{d}{d t} Z_{n}(t) \rightarrow 0, \quad n\left|Z_{n}(t)-Z(t)\right| \rightarrow 0 .
$$

Let $\eta \in C_{c}^{\infty}(0, T)$, and $\beta \in C_{c}^{\infty}\left(\mathbb{R}^{2}\right)$. We use the weak formulation of the adhesion equation with the test function $\psi_{n}(t, x)=\eta(t) \frac{1}{n^{2}} \beta\left(n\left(x-Z_{n}(t)\right)\right)$. We get

$$
\begin{array}{r}
\int_{0}^{T} \int_{\mathbb{R}^{2}}\left(\eta^{\prime}(t) \frac{1}{n^{2}} \beta\left(n\left(x-Z_{n}(t)\right)\right)+\eta(t) \frac{1}{n} \frac{d}{d t} Z_{n}(t) \cdot \nabla \beta\left(n\left(x-Z_{n}(t)\right)\right)\right) \\
\rho(t, x) d x d t \\
+\frac{1}{2} \sum_{i=1,2} \int_{0}^{T} \int_{\mathbb{R}^{4}} \eta(t) \frac{1}{n}\left(\frac{\partial}{\partial x_{i}} \beta\left(n\left(x-Z_{n}(t)\right)\right)-\frac{\partial}{\partial x_{i}} \beta\left(n\left(y-Z_{n}(t)\right)\right)\right) \\
-\frac{1}{4 \pi} \sum_{i, j=1,2} \int_{0}^{T} \int_{\mathbb{R}^{2}} \eta(t) \frac{\partial^{2}}{\partial x_{i} \partial x_{j}} \beta\left(n\left(x-Z_{n}(t)\right)\right) \nu_{i, j}(t, x) d x d t=0 .
\end{array}
$$

In view of (37), we can use dominated convergences to obtain in the limit $n \rightarrow \infty$

$$
\left.\sum_{i, j=1,2} \int_{0}^{T} \eta(t) \frac{\partial^{2}}{\partial x_{i} \partial x_{j}} \beta(0)\right) \nu_{i, j}(t,\{Z(t)\}) d t=0 .
$$

By choosing for instance $\beta(x)=|x|^{2}$ in a neighborhood of 0 , we deduce from the above equality that $\operatorname{tr}(\nu)$ does not charge $\Gamma$. Since $\nu$ is a non negative matrix valued measure, it is enough to conclude that $\nu$ does not charge $\Gamma$.

We prove now that there is always appearance of an atomic part in the solutions of the adhesion dynamics equation. We assume that $\int_{\mathbb{R}^{2}}|x|^{2} \rho_{I}(x) d x<\infty$. Then, 
as in the proof of Theorem 3.2, by using convenient truncation sequences, we prove that $\int_{\mathbb{R}^{2}}|x|^{2} \rho(t, x) d x$ is a bounded continuous function which satisfies in the sense of distribution

$$
\begin{aligned}
& \frac{d}{d t} \int_{\mathbb{R}^{2}}|x|^{2} \rho(t, x) d x \\
= & \sum_{i=1,2} \int_{\mathbb{R}^{4}}\left(x_{i}-y_{i}\right) K_{i}(x-y) \rho(t, x) \rho(t, y) d x d y \\
& -\frac{1}{2 \pi} \sum_{i=1,2} \int_{\mathbb{R}^{2}} \nu_{i, i}(t, x) d x \\
= & -\frac{1}{2 \pi}\left(\int_{\mathbb{R}^{4}}\left(1-\chi_{D}\right)(x, y) \rho(t, x) \rho(t, y) d x d y+\operatorname{tr}(\nu)\left(t, \mathbb{R}^{2}\right)\right) .
\end{aligned}
$$

We use (5) and the conservation law (25) to obtain

$$
\begin{aligned}
& \frac{d}{d t} \int_{\mathbb{R}^{2}}|x|^{2} \rho(t, x) d x \\
= & -\frac{1}{2 \pi}\left(\rho_{I}\left(\mathbb{R}^{2}\right)^{2}+\operatorname{tr}(\nu)\left(t, \mathbb{R}^{2}\right)-\sum_{a \in S_{a t}(\rho(t))} \rho(t,\{a\})^{2}\right) .
\end{aligned}
$$

The conservation of the first moment and (25) yields

$$
\frac{d}{d t} \int_{\mathbb{R}^{2}}\left|x-X_{I}\right|^{2} \rho(t, x) d x=\frac{d}{d t} \int_{\mathbb{R}^{2}}|x|^{2} \rho(t, x) d x .
$$

Therefore for any $t \in[0, T)$ we have

$$
\begin{aligned}
0 \leq & \int_{\mathbb{R}^{2}}\left|x-X_{I}\right|^{2} \rho(t, x) d x=\int_{\mathbb{R}^{2}}\left|x-X_{I}\right|^{2} \rho_{I}(x) d x \\
& -\frac{1}{2 \pi} \int_{0}^{t}\left(\rho_{I}\left(\mathbb{R}^{2}\right)^{2}+\operatorname{tr}(\nu)\left(s, \mathbb{R}^{2}\right)-\sum_{a \in S_{a t}(\rho(s))} \rho(s,\{a\})^{2}\right) d s .
\end{aligned}
$$

Let $T_{0}$ the maximal time such that for almost every $s \in\left[0, T_{0}\right], S_{a t}(\rho(s))=\emptyset$, we have

$$
\begin{aligned}
0 & \leq \int_{\mathbb{R}^{2}}\left|x-X_{I}\right|^{2} \rho_{I}(x) d x-\frac{1}{2 \pi} \int_{0}^{T_{0}}\left(\rho_{I}\left(\mathbb{R}^{2}\right)^{2}+\operatorname{tr}(\nu)\left(s, \mathbb{R}^{2}\right)\right) d s \\
& \leq \int_{\mathbb{R}^{2}}\left|x-X_{I}\right|^{2} \rho_{I}(x) d x-\frac{1}{2 \pi} T_{0} \rho_{I}\left(\mathbb{R}^{2}\right)^{2} .
\end{aligned}
$$

It gives the desired estimate on $T_{0}$.

We now study the asymptotic behavior for large time. Then we assume that $(\rho, \nu)$ is a global generalized solution. Since

$$
F(s)=\rho_{I}\left(\mathbb{R}^{2}\right)^{2}-\sum_{a \in S_{a t}(\rho(s))} \rho(s,\{a\})^{2}=\rho(s) \otimes \rho(s)\left(\mathbb{R}^{4} \backslash D\right)
$$

and $\operatorname{tr}(\nu)\left(s, \mathbb{R}^{2}\right)$ are non negative, the inequality (38) proves that these functions are integrable on $(0, \infty)$. Let $t_{n} \rightarrow \infty$, the measure $(s, x) \rightarrow \operatorname{tr}(\nu)\left(t_{n}+s, x\right)$ tends to 
0 tightly in $\mathcal{M}_{1}^{+}\left([0, \infty) \times \mathbb{R}^{2}\right)$ which leads to the first assertion on the asymptotic behavior. We also have that the function $s \rightarrow F\left(t_{n}+s\right)$ tends to 0 in $L^{1}(0, \infty)$. Therefore there is a subsequence $t_{n_{k}}$ such that $F\left(t_{n_{k}}+s\right) \rightarrow 0$, a.e. We use now the following Lemma whose proof is postponed at the end of this Section.

LEMMA 3.4. Let $\rho^{k} \in \mathcal{M}_{1}^{+}\left(\mathbb{R}^{D}\right), k=1,2, \ldots$ be a tightly bounded sequence of non negative measures such that $\rho^{k} \otimes \rho^{k}\left(\mathbb{R}^{2 D} \backslash D\right) \rightarrow 0$. Then, up to a subsequence, there exist $M \geq 0$ and $X \in \mathbb{R}^{2 D}$ such that $\rho^{k}(x) \rightarrow M \delta(x-X)$ tightly.

We apply this Lemma for $s$ a.e. to the sequence $\rho\left(t_{n_{k}}+s\right)$. Remark that the sequence is tightly bounded because $\int_{\mathbb{R}^{2}}|x|^{2} \rho\left(t_{n_{k}}+s, x\right) d x$ is uniformly bounded. Since the mass and the mean position of particles are conserved quantities we obtain that, up to subsequences, $\rho\left(t_{n_{k}}+s\right) \rightarrow \rho_{I}\left(\mathbb{R}^{2}\right) \delta\left(x-X_{I}\right)$. Since the limit is unique the whole sequence converges. But it follows from (30) that the sequence is equicontinuous with respect to time. Therefore $\rho\left(t_{n_{k}}+s\right) \rightarrow \rho_{I}\left(\mathbb{R}^{2}\right) \delta\left(x-X_{I}\right)$ for all $s \in[0, T)$. In particular $\rho\left(t_{n_{k}}\right) \rightarrow \rho_{I}\left(\mathbb{R}^{2}\right) \delta\left(x-X_{I}\right)$. Once again, the limit does not depend on the subsequence $t_{n_{k}}$ we have considered which proves that the convergence holds for the whole sequence. It concludes the proof of the Proposition.

Proof of Lemma 3.4. Let $\rho_{r e g}^{k}(x)=\rho^{k}(x)-\sum_{a \in S_{a t}\left(\rho^{k}\right)} \rho^{k}(\{a\}) \delta(x-a)$ be the regular part of the measure $\rho^{k}$. Since $0 \leq \rho_{\text {reg }}^{k} \leq \rho^{k}$, we have

$$
\rho_{r e g}^{k}\left(\mathbb{R}^{D}\right)^{2}=\rho_{r e g}^{k} \otimes \rho_{r e g}^{k}\left(\mathbb{R}^{2 D} \backslash D\right) \rightarrow 0 .
$$

Therefore $\rho_{\text {reg }}^{k} \rightarrow 0$ tightly and we can now assume without loss of generality that $\rho^{k}$ is purely atomic. Up to extracting a subsequence, for some $M \geq 0$, we have $M^{k}:=\rho^{k}\left(\mathbb{R}^{D}\right) \rightarrow M$. If $M=0$ the point $X$ can be chosen arbitrarily. We assume now $M>0$. We have

$$
\begin{aligned}
\rho^{k} \otimes \rho^{k}\left(\mathbb{R}^{2 D} \backslash D\right) & =\sum_{a \neq b \in S_{a t}\left(\rho^{k}\right)} \rho^{k}(\{a\}) \rho^{k}(\{b\}) \\
& =\sum_{a \in S_{a t}\left(\rho^{k}\right)} \rho^{k}(\{a\})\left(M^{k}-\rho^{k}(\{a\})\right) \rightarrow 0 .
\end{aligned}
$$

Let $N^{k}:=\sup _{a \in S_{a t}\left(\rho^{k}\right)} \rho^{k}(\{a\})$. We have $N^{k} \leq M^{k}$ and

$$
\begin{aligned}
M^{k}\left(M^{k}-N^{k}\right) & =\sum_{a \in S_{a t}\left(\rho^{k}\right)} \rho^{k}(\{a\})\left(M^{k}-N^{k}\right) \\
& \leq \sum_{a \in S_{a t}\left(\rho^{k}\right)} \rho^{k}(\{a\})\left(M^{k}-\rho^{k}(\{a\})\right) \rightarrow 0 .
\end{aligned}
$$

We conclude that $N^{k} \rightarrow M>0$. Therefore for $k$ large enough there exist $X^{k}$ such that $\rho^{k}\left(\left\{X^{k}\right\}\right) \geq \frac{4}{5} N_{k} \geq \frac{3}{5} M>M_{k} / 2$. Then all other atoms $a$ satisfy $\rho^{k}(\{a\})<M_{k} / 2$. It yields $N_{k}=\max _{a \in S_{a t}\left(\rho^{k}\right)} \rho^{k}(\{a\})=\rho^{k}\left(\left\{X^{k}\right\}\right) \rightarrow M$. Since the sequence is tightly bounded we obtain also that the sequence $X^{k}$ is bounded. Still up to a subsequence, we thus can assume that $X^{k} \rightarrow X$. We have $\rho^{k}(x)-\rho^{k}\left(\left\{X^{k}\right\}\right) \delta\left(x-X^{k}\right) \rightarrow 0$ and $\rho^{k}\left(\left\{X^{k}\right\}\right) \delta\left(x-X^{k}\right) \rightarrow M \delta(x-X)$ tightly. It ends the proof of the Lemma and the Section. 
4. Euler equation with vortex points of distinguished sign. This Section is concerned with the existence of weak solutions of the two dimensional Euler equations with vortex points initial data. The Euler equations read

$$
\begin{array}{r}
\frac{\partial}{\partial t} u_{i}+\sum_{j=1}^{2} \frac{\partial}{\partial x_{j}}\left(u_{j} u_{i}\right)+\frac{\partial}{\partial x_{i}} p=0, \quad t \in \mathbb{R}, x \in \mathbb{R}^{2}, \quad i=1,2 \\
\sum_{j=1}^{2} \frac{\partial}{\partial x_{j}}\left(u_{j}\right)=0 \quad t \in \mathbb{R}, x \in \mathbb{R}^{2} .
\end{array}
$$

The vorticity is defined by $\omega=\frac{\partial}{\partial x_{1}} u_{2}-\frac{\partial}{\partial x_{2}} u_{1}$ and we deduce immediately from (39) that it is advected by the velocity $u=\left(u_{1}, u_{2}\right)$. We have

$$
\begin{array}{r}
\frac{\partial}{\partial t} \omega+\sum_{j=1}^{2} \frac{\partial}{\partial x_{j}}\left(u_{j} \omega\right)=0, \quad t \in \mathbb{R}, x \in \mathbb{R}^{2}, \\
\omega=\frac{\partial}{\partial x_{1}} u_{2}-\frac{\partial}{\partial x_{2}} u_{1}, \quad \sum_{j=1}^{2} \frac{\partial}{\partial x_{j}}\left(u_{j}\right)=0 \quad t \in \mathbb{R}, x \in \mathbb{R}^{2} .
\end{array}
$$

We study the case where $\omega$ is a bounded measure. The case which has been intensively studied is the case of vortex sheets which correspond to concentrations of the measure $\omega$ on surfaces in time space (lines in the 2D-space). vortex points correspond to concentrations on curves in time space (points in the 2D-space). To obtain a global existence result of weak solutions the difficulty comes from the non-linear terms $u_{j} u_{i}$ in (39) or $u_{j} \omega$ in (41). Starting from smooth global solutions, $u^{n}$, which are known to exist, the goal is to pass to the limit in the terms $u_{j}^{n} u_{i}^{n}$. Actually it was pointed out by Di Perna and Majda, see [12], that it is necessary to pass to the limit only in the special non linearity $\left(u_{1}^{n}\right)^{2}-\left(u_{2}^{n}\right)^{2}$ and $u_{1}^{n} u_{2}^{n}$. However when the vorticity is only a bounded measure and not better there is a lack of compactness in $L_{l o c}^{2}\left(\mathbb{R}^{2}\right)$ for the sequence $u^{n}$ which is a major obstruction to the fact that $\lim _{n \rightarrow \infty} u^{n} \otimes u^{n}=\lim _{n \rightarrow \infty} u^{n} \otimes \lim _{n \rightarrow \infty} u^{n}$. One way to study such a lack of compactness is to introduce a defect measure related to the sequence $u^{n}$. Such an approach has been followed by many authors, see $[1,11,31]$. In particular situations (concentration-cancellation), it is possible to conclude that the defect measure vanishes, leading to an existence theorem. The next step in the theory has been performed by Delort in [9]. He proved that if a sequence $u_{n}$ is bounded in $\left[L_{l o c}^{2}\left(\mathbb{R}^{2}\right)\right]^{2}$, is divergence free and has a vorticity with a distinguished sign bounded in $\mathcal{M}\left(\mathbb{R}^{2}\right)$ then the special non linearities $\left(u_{1}^{n}\right)^{2}-\left(u_{2}^{n}\right)^{2}$ and $u_{1}^{n} u_{2}^{n}$ pass to the limit even if the complete matrix $u^{n} \otimes u^{n}$ does not. It allows to obtain a global in time existence result of weak solution for vortex sheets of prescribed sign. Let us remark that the vorticity $\omega^{n}$ is bounded in $H_{l o c}^{-1}\left(\mathbb{R}^{2}\right)$ in this context. It allows Delort to prove that there is no point concentration. Therefore Delort existence theorem do not include the case of vortex points. Our goal is to use the techniques developed in the previous Section to study weak solution with vortex points of distinguished sign. Remark that in this case $u(t)$ is no more in $L_{l o c}^{2}\left(\mathbb{R}^{2}\right)$ which poses the problem of the definition of the product $u \otimes u$. We conclude the introduction of this Section by pointing out that the existence of weak solution of vortex sheets with no distinguished sign is still an outstanding open problem. The only result we know in this direction is due to M.C. 
Lopes Filho, H.J. Nussenzveig Lopes, Zhouping Xin, [23]. It is an existence result when the non negative and the non positive part of vortex sheets are separated by a symmetry axis. We now introduce the weak vorticity formulation of Euler equation which is one of the key ingredient in Delort's proof.

Let $x=\left(x_{1}, x_{2}\right) \in \mathbb{R}^{2}$ be any vector, we define $x^{\perp}=\left(-x_{2}, x_{1}\right)$. The equations (42) supplemented by convenient conditions of decreasing of the velocity field at infinity are equivalent to the Biot-Savart law which expresses the velocity in term of the vorticity

$$
u(t, x)=\frac{1}{2 \pi} \int_{\mathbb{R}^{2}} \frac{(x-y)^{\perp}}{|x-y|^{2}} \omega(t, y) d y
$$

Using the skew property of the kernel $\frac{(x)^{\perp}}{|x|^{2}}$, the equations (41) and (42) lead to the following weak vorticity formulation

$$
\begin{array}{r}
\forall \varphi \in C_{c}^{\infty}\left((-\infty, \infty) \times \mathbb{R}^{2}\right), \quad \int_{-\infty}^{\infty} \int_{\mathbb{R}^{2}} \frac{\partial}{\partial t} \varphi \omega(t, x) d x d t= \\
-\frac{1}{4 \pi} \int_{-\infty}^{\infty} \int_{\mathbb{R}^{4}} H_{\varphi(t)}(x, y) \omega(t, x) \omega(t, y) d x d y d t
\end{array}
$$

where the function $H_{\varphi(t)}$ is defined as follows.

$$
\forall \psi \in C^{1}\left(\mathbb{R}^{2}\right), \quad H_{\psi}(x, y)=\frac{(x-y)^{\perp}(\nabla \psi(x)-\nabla \psi(y))}{|x-y|^{2}}
$$

The function $H_{\varphi(t)}$ is bounded, tends to 0 at infinity and is continuous except on the diagonal. If $\omega^{n}(t)$ is a non negative sequence, vaguely equicontinuous with respect to time and has no concentration point then the non-linear term

$$
\int_{-\infty}^{\infty} \int_{\mathbb{R}^{4}} H_{\varphi(t)}(x, y) \omega^{n}(t, x) \omega^{n}(t, y) d x d y d t
$$

is under control. When the velocity $\omega^{n}(t)$ is uniformly with respect to $n$ and $t$ bounded in $H_{l o c}^{-1}\left(\mathbb{R}^{2}\right)$, Delort proved that point concentration cannot occur which leads to his existence theorem. Majda, in [24], proved a quantitative estimate for this no concentration effect. We refer for more details on this subject to the original Delort works, [9], to [14, 24] or to the enlightening presentation given by Schochet in [29]. We want to relax the condition $\omega_{I} \in H_{l o c}^{-1}\left(\mathbb{R}^{2}\right)$ in order to consider vortex points. The price to pay is, as in the previous Section, the introduction of a diagonal defect measure for the sequence $\omega^{n}(t) \otimes \omega^{n}(t)$. Let us remark that the diagonal defect measure is, in a way, a micro-localization of the defect measure related to the sequence $u^{n}(t)$. As in the previous Section we have a generalization of the product $\omega u$.

Definition 4.8. Let $I$ be a real interval, let $(\omega, \nu) \in \mathcal{D} \mathcal{M}^{+}\left(I ; \mathbb{R}^{2}\right)$, then the vortex flux $q(\omega, \nu)=\left(q_{1}(\omega, \nu), q_{2}(\omega, \nu)\right)$ is defined by $q(\omega, \nu)=j(\omega, \nu)^{\perp}$ where $j$ is given in Definition 3.6. 
In other words we have

$$
\begin{array}{r}
\forall \psi \in C_{c}^{1}\left(I \times \mathbb{R}^{2}\right), \quad \int_{I} \int_{\mathbb{R}^{2}} \psi(t, x) q(\omega, \nu)(t, x) d x d t= \\
\frac{1}{4 \pi} \int_{I} \int_{\mathbb{R}^{4} \backslash D} \frac{(x-y)^{\perp}(\psi(t, x)-\psi(t, y))}{|x-y|^{2}} \omega(t, x) \omega(t, y) d x d y d t \\
-\frac{1}{4 \pi} \int_{I} \int_{\mathbb{R}^{2}}(\nu(t, x) \cdot \nabla \psi(t, x))^{\perp} d x d t .
\end{array}
$$

Remark that the first integral is out of the diagonal. As in the previous Section this definition of the vortex flux corresponds to a concept of generalized solutions of the weak vorticity formulation of Euler equations.

Definition 4.9. Let $T \in(0,+\infty]$, a couple $(\omega, \nu) \in \mathcal{D M}^{+}\left((-T, T) ; \mathbb{R}^{2}\right)$ is a generalized solution on $(-T, T)$ of Euler equation in weak vorticity form with the Cauchy data $\omega_{I} \in \mathcal{M}_{1}^{+}\left(\mathbb{R}^{2}\right)$ if and only if $\omega(0)=\omega_{I}$ and

$$
\frac{\partial}{\partial t} \omega+\sum_{i=1,2} \frac{\partial}{\partial x_{i}} q_{i}(\omega, \nu)=0
$$

in the sense of distribution on $(-T, T) \times \mathbb{R}^{2}$.

Let us remark that if there is no atomic part to the measures $\omega(t), t \in(-T, T)$ then the defect measure vanishes and $\omega$ is a weak solution of the classical formulation (44). The generalized solutions also satisfy the classical conservation law and are stable for the weak convergence of measures.

THEOREM 4.3. We have the following results concerning generalized solutions. Global existence. For all initial data $\omega_{I} \in \mathcal{M}_{1}^{+}\left(\mathbb{R}^{2}\right)$, there is a generalized solution on $(-\infty, \infty)$.

A priori estimates. Let $T \in(0,+\infty]$, and let $(\omega, \nu) \in \mathcal{D} \mathcal{M}^{+}\left((-T, T) ; \mathbb{R}^{2}\right)$ be a generalized solution on $(-T, T)$ of Euler equation in weak vorticity form, with the Cauchy data $\omega_{I} \in \mathcal{M}_{1}^{+}\left(\mathbb{R}^{2}\right)$. Then we have the conservation law

$$
\forall t \in(-T, T), \quad \omega\left(t, \mathbb{R}^{2}\right)=\omega_{I}\left(\mathbb{R}^{2}\right) .
$$

Moreover, we have an a priori tight bound of the solution. There is a universal constant, $C>0$, such that

$$
\forall R>0, \forall t \in(-T, T), \quad \omega\left(t, \mathbb{R}^{2} \backslash B_{R}\right) \leq \omega_{I}\left(\mathbb{R}^{2} \backslash B_{R / 2}\right)+C \frac{|t|}{R^{2}} \omega_{I}\left(\mathbb{R}^{2}\right)^{2}
$$

If $|x|^{p} \omega_{I}$ with $p=1$ or 2 , is assumed to be a bounded measure then the same is true for $|x|^{p} \omega(t), t \in(-T, T)$ and there is a universal constant $C>0$ such that

$$
\begin{aligned}
\forall t & \in(-T, T), \int_{\mathbb{R}^{2}}|x|^{p} \omega(t, x) d x \leq \int_{\mathbb{R}^{2}}|x|^{p} \omega_{I}(x) d x \\
& +C \omega_{I}\left(\mathbb{R}^{2}\right)\left(1+|t| \omega_{I}\left(\mathbb{R}^{2}\right)\right) .
\end{aligned}
$$

In this case the first moment is a conserved quantity

$$
\forall t \in(-T, T), \quad \int_{\mathbb{R}^{2}} x \omega(t, x) d x=\int_{\mathbb{R}^{2}} x \omega_{I}(x) d x .
$$


If $p=2$ we have moreover conservation of the second moment

$$
\forall t \in(-T, T), \quad \int_{\mathbb{R}^{2}}|x|^{2} \omega(t, x) d x=\int_{\mathbb{R}^{2}}|x|^{2} \omega_{I}(x) d x .
$$

The equicontinuity of generalized solutions can be estimated as follows. For every test function $\psi \in C_{b}^{2}\left(\mathbb{R}^{2}\right)$, there is a positive constant $C(\psi)$ which depends only on the test function such that

$$
\left|\frac{d}{d t} \int_{\mathbb{R}^{2}} \psi(x) \omega(t, x) d x\right| \leq C(\psi) \omega_{I}\left(\mathbb{R}^{2}\right)^{2}
$$

in the distribution sense on $(-T, T)$.

It follows that if $\left(\omega^{n}, \nu^{n}\right) \in \mathcal{D} \mathcal{M}^{+}\left((-T, T) ; \mathbb{R}^{2}\right), n=1,2, \ldots$ is a sequence of generalized solutions such that $\omega_{I}^{n}=\omega^{n}(t=0)$ is tightly bounded then the sequence $\omega^{n}(t), t \in(-T, T)$ is locally tightly uniformly bounded and equicontinuous with respect to time.

Stability. The set of generalized solutions is stable. More precisely let $\left(\omega^{n}, \nu^{n}\right) \in$

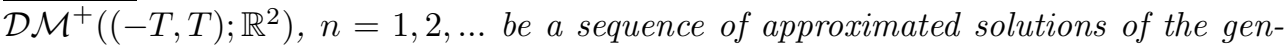
eralized weak vorticity formulation:

$$
\frac{\partial}{\partial t} \omega^{n}+\sum_{i=1,2} \frac{\partial}{\partial x_{i}} q_{i}\left(\omega^{n}, \nu^{n}\right)=S^{n}
$$

with $S^{n} \rightarrow 0$ in the sense of distribution on $(0, T) \times \mathbb{R}^{2}$ as $n \rightarrow \infty$. We assume that the sequence of measures $\left(\omega^{n}(t)\right)_{n \geq 1}$ is locally tightly uniformly bounded and equicontinuous with respect to time. Then all accumulation points of the sequence $\left(\omega^{n}, \nu^{n}\right)$ for the convergence in diagonal defect measures obtained by Corollary 2.3 are generalized solutions of the vorticity formulation of Euler equations.

Proof. The main points in the proof are mutatis mutandis the ones of the proof of Theorem 3.2. Therefore we only point out the differences.

The first difference comes from the fact that global smooth solutions of Euler equation are known to exist. Therefore the global existence result is a consequence of the stability statement and of the a priori bounds.

The stability statement is still a consequence of Lemma 3.3.

To obtain the a priori bounds we remark that in the sense of distribution on $(-T, T)$ we have

$$
\begin{aligned}
& \frac{d}{d t} \int_{\mathbb{R}^{2}} \psi(x) \omega(t, x) d x \\
= & \frac{1}{4 \pi} \int_{\mathbb{R}^{4} \backslash D} \frac{(x-y)^{\perp} \cdot(\nabla \psi(x)-\nabla \psi(y))}{|x-y|^{2}} \omega(t, x) \omega(t, y) d x d y \\
& +\frac{1}{4 \pi} \int_{\mathbb{R}^{2}} \frac{\partial^{2}}{\partial x_{1} \partial x_{2}} \psi(x)\left(\nu_{1,1}-\nu_{2,2}\right)(t, x) d x \\
& +\frac{1}{4 \pi} \int_{\mathbb{R}^{2}}\left(\frac{\partial^{2}}{\partial x_{2}{ }^{2}} \psi(x)-\frac{\partial^{2}}{\partial x_{1}{ }^{2}} \psi(x)\right) \nu_{1,2}(t, x) d x .
\end{aligned}
$$

Thus we obtain the analogue of the estimate (31). The remainder of the proof is exactly the same. The conservation of the second moment comes from the fact that 
the right hand side of the above equation vanishes for $\psi(x)=|x|^{2}$.

The null sets of the defect measure can also be characterized. Exactly in the same way as in Section 3 we prove

Proposition 4.3. Let $(\omega, \nu) \in \mathcal{D} \mathcal{M}^{+}\left((-T, T) ; \mathbb{R}^{2}\right)$ be a generalized solution. Let $S=\left\{(t, a) / t \in(-T, T), a \in S_{a t}(\omega(t))\right\}$ be the singular support of the measure $\omega$. If $\Gamma$ is a Borelian set which does not meet $S(\Gamma \cap S=\emptyset)$ or if $\Gamma$ is an Hölder continuous curve, $\Gamma=\left\{(t, Z(t)) / t \in(-T, T), Z \in C^{0, \gamma}\left(\mathbb{R} ; \mathbb{R}^{2}\right\}\right\}$, with coefficient $\gamma>1 / 2$ then the defect measure $\nu$ does not charge $\Gamma$ :

$$
\int_{\Gamma} \nu(t, x) d x d t=0
$$

In the theory of Delort and Schochet, $[9,29]$, it is possible to consider an initial vorticity with integrable non positive parts, $\omega_{I} \in\left(\mathcal{M}_{1}^{+}\left(\mathbb{R}^{2}\right)+L^{1}\left(\mathbb{R}^{2}\right)\right) \cap H_{\text {loc }}^{-1}\left(\mathbb{R}^{2}\right)$. One difficulty to obtain such a result in the present context is that we do not know if the positive part of a sequence of smooth solutions are equicontinuous with respect to time. A remedy is to change the definition of diagonal defect measures to take into account sequences of measures with non positive parts in a compact set of $L^{1}-$ weak. In the sake of concision, we do not follow this approach and we prefer to give a result about perturbation in $L^{p}$ for $p>2$.

TheOREM 4.4. Let $p \in(2, \infty]$. For all initial data $\omega_{I} \in \mathcal{M}_{1}^{+}\left(\mathbb{R}^{2}\right)+\left(L^{1} \cap L^{p}\left(\mathbb{R}^{2}\right)\right)$ there exist $\left(\omega^{+}, \nu\right) \in \mathcal{D} \mathcal{M}^{+}\left((-\infty, \infty) ; \mathbb{R}^{2}\right)$ and $\omega^{r} \in C^{0}\left((-\infty, \infty) ; L^{1} \cap L^{p}\left(\mathbb{R}^{2}\right)\right.$-weak $)$ such that $\omega=\omega^{+}+\omega^{r}$ is a solution of Euler equation, corresponding to the initial data $\omega_{I}=\omega(t=0)$, in the following generalized weak vorticity formulation

$$
\begin{aligned}
& \forall \psi \in C_{c}^{\infty}\left((-\infty, \infty) \times \mathbb{R}^{2}\right), \quad \int_{-\infty}^{\infty} \int_{\mathbb{R}^{2}} \frac{\partial}{\partial t} \psi(t, x) \omega(t, x) d x d t \\
& +\frac{1}{4 \pi} \int_{-\infty}^{\infty} \int_{\mathbb{R}^{4} \backslash D} \frac{(x-y)^{\perp} \cdot(\nabla \psi(t, x)-\nabla \psi(t, y))}{|x-y|^{2}} \omega(t, x) \omega(t, y) d x d y d t \\
& +\frac{1}{4 \pi} \int_{-\infty}^{\infty} \int_{\mathbb{R}^{2}} \frac{\partial^{2}}{\partial x_{1} \partial x_{2}} \psi(t, x)\left(\nu_{2,2}-\nu_{1,1}\right)(t, x) d x d t \\
& +\frac{1}{4 \pi} \int_{-\infty}^{\infty} \int_{\mathbb{R}^{2}}\left(\frac{\partial^{2}}{\partial x_{1}{ }^{2}} \psi(t, x)-\frac{\partial^{2}}{\partial x_{2}{ }^{2}} \psi(t, x)\right) \nu_{1,2}(t, x) d x d t=0 .
\end{aligned}
$$

Proof. Let us consider two regularized sequence of smooth initial data $\left(\omega_{I}^{n,+}\right)_{n \geq 1}$ and $\left(\omega_{I}^{n, r}\right)_{n \geq 1}$ such that

$$
\begin{array}{r}
\omega_{I}^{n,+} \geq 0, \quad \omega_{I}^{n,+} \rightarrow \omega_{I}^{+} \text {tightly }, \\
\omega_{I}^{n, r} \rightarrow \omega_{I}^{r} \text { in } L^{1} \cap L^{p}\left(\mathbb{R}^{2}\right) .
\end{array}
$$

The vorticity $\omega^{n}$ solution of the Euler equation can be decomposed in $\omega^{n}=\omega^{n,+}+\omega^{n, r}$ corresponding to the initial data $\omega_{I}^{n}=\omega_{I}^{n,+}+\omega_{I}^{n, r}$. The functions $\omega^{n,+}(t)$ (respectively $\left.\omega^{n, r}(t)\right)$ are uniformly bounded with respect to $n$ and $t$ in $L^{1}\left(\mathbb{R}^{2}\right)$ (respectively in $\left.L^{1} \cap L^{p}\left(\mathbb{R}^{2}\right)\right)$. Let us split the velocity in $u^{n}=u^{n,+}+u^{n, r}$. Classical results in harmonic analysis show that $\left(u^{n, r}(t)\right)_{n \geq 1, t \in \mathbb{R}}$ is bounded in $C_{b}^{0}\left(\mathbb{R}^{2}\right)$ and lies in a compact of $C_{b}^{0}(K)$ for every compact subset $K \subset \mathbb{R}^{2}$. We have

$$
\frac{\partial}{\partial t} \omega^{n,+}(t)+\operatorname{div}\left(q\left(\omega^{n,+}, 0\right)+u^{n, r}(t) \omega^{n,+}\right)=0
$$


which shows that $\frac{\partial}{\partial t} \omega^{n,+}(t)$ is uniformly with respect to $t$ and $n$, bounded in the distributions space of $\mathbb{R}^{2}$. As in the proof of Theorem 3.2 we also deduce from the above equation that for every $T>0, \omega^{n,+}(t), n \geq 1, t \in[-T, T]$, is tightly bounded. From this two facts, we conclude that $\omega^{n,+}$ is uniformly tightly bounded and equicontinuous with respect to $t \in[-T, T]$. By Corollary 2.3 and Lemma 3.3, we obtain that up to a subsequence $\left(\omega^{n,+}, 0\right) \rightarrow\left(\omega^{+}, \nu\right) d . m$. and $q\left(\omega^{n,+}, 0\right) \rightarrow q\left(\omega^{+}, \nu\right)$ in the sense of distribution on $(-\infty, \infty) \times \mathbb{R}^{2}$. For the regular part we have

$$
\frac{\partial}{\partial t} \omega^{n, r}(t)+\operatorname{div}\left(\left(u^{n,+}(t)+u^{n, r}(t)\right) \omega^{n, r}\right)=0 .
$$

The $L^{1}$-bound of $\omega^{n,+}(t)$ implies that $u^{n,+}(t)$ is uniformly bounded in $L^{1} \cap L^{2-\alpha}\left(\mathbb{R}^{2}\right)+$ $L^{2+\alpha} \cap L^{\infty}\left(\mathbb{R}^{2}\right)$ for every $\alpha>0$. Therefore $u^{n}(t) \omega^{n, r}(t)$ is uniformly bounded in $L^{1}\left(\mathbb{R}^{2}\right)$. As before we deduce that $\frac{\partial}{\partial t} \omega^{n, r}(t)$ is uniformly with respect to $t$ and $n$, bounded in the distributions space of $\mathbb{R}^{2}$ and that $\omega^{n, r}(t), n \geq 1, t \in[-T, T]$, is tightly bounded. Standard arguments show that up to a subsequence $\omega^{n, r} \rightarrow \omega^{r}$ in $C^{0}\left([-T, T] ; L^{1} \cap L^{p}\left(\mathbb{R}^{2}\right)-\right.$ weak $)$. Since the map from $L^{1} \cap L^{p}\left(\mathbb{R}^{2}\right)$ to $C_{b}^{0}(K)$ : $\omega \mapsto u=\frac{x^{\perp}}{|x|^{2}} * \omega$, is compact, we have $u^{n, r} \rightarrow u^{r}$ in $C_{b}^{0}([-T, T] \times K)$ for every compact subset $K \subset \mathbb{R}^{2}$. Thanks to the tight bound of $\omega^{n,+}(t)$ and the uniform bound in $C_{b}^{0}\left([-T, T] \times \mathbb{R}^{2}\right)$ of $u^{n, r}$ we can now pass to the limit in (52) to obtain

$$
\frac{\partial}{\partial t} \omega^{+}(t)+\operatorname{div}\left(q\left(\omega^{+}, \nu\right)+u^{r}(t) \omega^{+}(t)\right)=0, \quad \omega^{+}(0)=\omega_{I}^{+}
$$

It remains to pass to the limit in the term $u^{n,+}(t) \omega^{n, r}(t)$ in $(53)$. Let $\psi \in C_{c}^{\infty}\left(\mathbb{R}^{2}\right)$. We have

$$
\begin{aligned}
& \int_{\mathbb{R}^{2}} u^{n,+}(t) \omega^{n, r}(t) \psi(x) d x \\
= & \int_{\mathbb{R}^{4}} \frac{(x-y)^{\perp}}{2 \pi|x-y|^{2}} \omega^{n, r}(t, x) \psi(x) \omega^{n,+}(t, y) d x d y
\end{aligned}
$$

Exactly for the same reasons as for the functions $u^{n, r}$, the sequence of functions

$$
y \mapsto \int_{\mathbb{R}^{2}} \frac{(x-y)^{\perp}}{2 \pi|x-y|^{2}} \omega^{n, r}(t, x) \psi(x) d x
$$

converges in $C_{b}^{0}([-T, T] \times K)$ and is bounded in $C_{b}^{0}\left([-T, T] \times \mathbb{R}^{2}\right)$ for every $T>0$ and for every compact subset $K \subset \mathbb{R}^{2}$. Therefore

$$
\int_{\mathbb{R}^{2}} u^{n,+}(t) \omega^{n, r}(t) \psi(x) d x \rightarrow \int_{\mathbb{R}^{4}} \frac{(x-y)^{\perp}}{2 \pi|x-y|^{2}} \omega^{r}(t, x) \psi(x) \omega^{+}(t, y) d x d y
$$

uniformly with respect to $t \in[-T, T]$. Therefore we can pass to the limit in (53) to obtain

$$
\frac{\partial}{\partial t} \omega^{r}(t)+\operatorname{div}\left(\left(u^{+}(t)+u^{r}(t)\right) \omega^{n, r}\right)=0
$$

The weak vorticity formulation of the theorem is obtained by combining (55) and (54). Remark that the formulation (55) and (54) is actually more precise but destroy in a way the symmetry of the vorticity formulation. It is the price to pay to obtain the continuity with respect to time of the singular non negative part of the vorticity. 


\section{REFERENCES}

[1] Alinhac, S., Un phénomène de concentration évanescente pour des flots non-stationnaires incompressibles en dimension deux, Comm. Math. Phys., 127:3 (1990), pp. 585-596.

[2] Bouchut F., James F., Duality solutions for pressure-less gases, monotone scalar conservation laws, and uniqueness, Comm. Partial Differential Equations, 24:11-12 (1999), pp. 21732189 .

[3] Bourbaki N., Livre VI, Intégration, Chapitre IX, Ed. Hermann, (Paris) 1969.

[4] BRenier Y., Convergence of the Vlasov-Poisson system to the incompressible Euler equations., Comm. in Partial Diff. Eq., 25:3-4 (2000), pp. 737-754.

[5] Brenier Y., Personal communication.

[6] Brenier Y., Grenier E., Sticky particles and scalar conservation laws, SIAM J. Numer. Anal., 35:6 (1998), pp. 2317-2328.

[7] Chemin J.-Y., Perfect incompressible fluids, Oxford Lecture Series in Mathematics and its Applications, 14. The Clarendon Press, Oxford University Press, New York, 1998.

[8] Constantin P., Majda A. J., Tabak E., Formation of Strong Fronts in the $2-$ D Quasigeostrophic Thermal Active Scalar, Nonlinearity, 7 (1994), pp. 1945-1533.

[9] Delort, J.-M., Existence de nappes de tourbillon en dimension deux (French), J. Amer. Math. Soc., 4:3 (1991), pp. 553-586.

[10] Dermoune, A., Probabilistic interpretation of sticky particle model, Ann. Probab., 27:3 (1999), pp. 1357-1367.

[11] DiPerna, R. J.; Majda, A. J., Concentrations in regularizations for 2-D incompressible flow, Comm. Pure Appl. Math., 40:3 (1987), pp. 301-345.

[12] DiPerna, R. J., MAJdA, A. J., Reduced Hausdorff dimension and concentration-cancellation for two-dimensional incompressible flow, J. Amer. Math. Soc., 1:1 (1988), pp. 59-95.

[13] E W., Rykov Y., SinAi Y. G., Generalized variational principles, global weak solutions and behavior with random initial data for systems of conservation laws arising in adhesion particle dynamics, Comm. Math. Phys., 177:2 (1996), pp. 349-380.

[14] GÉRARD P., Résultats récents sur les fluides parfaits incompressibles bidimensionnels d'après J.-Y. Chemin et J.-M. Delort, Astérisque, 206 (1992), pp. 411-444.

[15] Golse F., Saint-Raymond L., The Vlasov-Poisson system with strong magnetic field., J. Math. Pures Appl., 78 (1999), pp. 798-817.

[16] Grenier, E., Pseudo-differential energy estimates of singular perturbations, Comm. Pure Appl. Math., 50:9 (1997), pp. 821-865.

[17] Herrero M. A., Medina B., Velázquez J. J. L., Self-similar Blow-up for a ReactionDiffusion System, J. Comp. App. Math., 97 (1998), pp. 99-119.

[18] Herrero M. A., Velázquez J. J. L., Chemotactic collapse for the Keller-Segel model, J. Math. Biol., 35 (1996), pp. 176-194.

[19] Jabin P. E., Macroscopic limit of Vlasov type equations with friction, Annales I.H.P., Anal. non linéaire, 7:5 (2000), pp. 651-672.

[20] JäGER W., LuCKhaus S., On explosions of solutions to a system of partial differential equations modelling chemotaxis, Trans. A. M. S., 329:2 (1992), pp. 819-824.

[21] Keller E.F., Segel L.A., Initiation of slime mold aggregation view as an instability, J. Theor. Biol., 26 (1970), pp. 399-415.

[22] Lions P.-L., Mathematical topics in fluid mechanics. Vol. 1. Incompressible models, Oxford Lecture Series in Mathematics and its Applications no 3, Oxford Science Publications, The Clarendon Press, Oxford University Press, New York, 1996.

[23] Lopes Filho M.C., Nussenzveig Lopes H. J., Xin Z., Existence of Vortex Sheets with Reflection Symmetry in Two Space Dimensions, Arch. Rational Mech. Anal., 158:3 (2001), pp. 235-257.

[24] MAJDA A. J., Remarks on weak solutions for vortex sheets with a distinguished sign, Indiana Univ. Math. J., 42:3 (1993), pp. 921-939.

[25] Marchioro C., Pulvirenti M., Mathematical theory of incompressible non viscous fluids, Applied Mathematical Sciences, Vol. 96, Springer-Verlag, New York Berlin Heidelberg, 1994.

[26] Nieto J., Poupaud F., Soler J., High field limit for Vlasov-Poisson-Fokker-Planck equations, Arch. Rational Mech. Anal., 158:1 (2001), pp. 29-59.

[27] Poupaud F., Runaway phenomena and fluid approximation under high fields in semiconductor kinetic theory, Z. Angew. Math. Mech., 72 (1992), pp. 359-372.

[28] Rascle M., Ziti C., Finite time blow-up in some models of chemotaxis, J. Math. Biology, 33 (1995), pp. 388-414

[29] Schochet, S., The weak vorticity formulation of the 2D Euler equations and concentration- 
cancellation, Comm. in PDE, 20 (1995), pp. 1077-1104.

[30] Schwartz, L., Cours d'analyse Vol. 1, Hermann, Paris, 1981.

[31] Zheng, Y., Concentration-cancellation for the velocity fields in two-dimensional incompressible fluid flows, Comm. Math. Phys., 135:3 (1991), pp. 581-594.

[32] Ziтi C., Problème hyperboliques non linéaires en dynamique des populations chimiotactiques, Thèse d'Etat es-sciences, Faculté des Sciences de Fes, Maroc, 1996. 
F. POUPAUD 